TITLE:

\title{
Super- and subcritical hydration of nonpolar solutes. I. Thermodynamics of hydration
}

$\operatorname{AUTHOR}(S)$ :

Matubayasi, N; Nakahara, M

\section{CITATION:}

Matubayasi, N ... [et al]. Super-and subcritical hydration of nonpolar solutes. I.

Thermodynamics of hydration. JOURNAL OF CHEMICAL PHYSICS 2000, 112 (18): 8089-8109

\section{ISSUE DATE:}

2000-05-08

URL:

http://hdl.handle.net/2433/50350

\section{RIGHT:}

Copyright 2000 American Institute of Physics. This article may be downloaded for personal use only. Any other use requires prior permission of the author and the American Institute of Physics. 


\title{
Super- and subcritical hydration of nonpolar solutes. I. Thermodynamics of hydration
}

\author{
Nobuyuki Matubayasi and Masaru Nakahara \\ Institute for Chemical Research, Kyoto University, Uji, Kyoto 611-0011, Japan
}

(Received 13 September 1999; accepted 14 February 2000)

\begin{abstract}
Hydration thermodynamics of nonpolar solutes in high-temperature water is investigated by computer simulations. The excess chemical potentials of the methane and hard sphere solutes are evaluated over a wide range of density and temperature, and the thermodynamic origin of the enhanced affinity of the nonpolar solute for super- and subcritical water is identified. It is shown that when the density is medium to high in the high-temperature conditions, the enhanced affinity results from the elevated temperature and represents the nonspecific aspect of super- and subcritical water. The excess chemical potentials are further decomposed into the enthalpic and entropic components. It is found that when the system is moved from the ambient state to a high-temperature state, the accompanying change is unfavorable for the enthalpic component and is favorable for the entropic component. The thermodynamics of cavity formation is also pursued in connection to the size distribution of cavities in pure solvent water. The utility of the scaled-particle theory is then demonstrated over a wide range of thermodynamic conditions, and the effective diameter of the water molecule is assigned within the framework of the scaled-particle theory. (C) 2000 American Institute of Physics. [S0021-9606(00)50418-8]
\end{abstract}

\section{INTRODUCTION}

Oil does not mix with water. This is a common sense at ambient conditions. In supercritical conditions, however, a reverse property is observed. Oil dissolves well into supercritical water. ${ }^{1-8}$ In other words, while a nonpolar solute is sparsely soluble in water at ambient conditions, it is no more "hydrophobic" at supercritical conditions. Due to this remarkable property, supercritical water serves as a novel medium for organic chemical reactions which are often of environmental and industrial importance. ${ }^{8-12}$ In determining the equilibrium constants and reaction rates of the chemical reactions, the role of hydration cannot be overemphasized. Furthermore, the availability of a wide range of density and temperature is the most useful characteristic of supercritical water, and a large variation in the density and temperature may lead to a drastic change in the hydration effect on chemical reactions. Thus, in order to understand and control the organic chemical reactions in supercritical water, it is essential to establish a molecular picture of hydration of nonpolar solutes over a wide range of thermodynamic conditions.

The study of supercritical water as a pure solvent system forms a basis for addressing aqueous solutions at supercritical states. In previous papers, ${ }^{13-15}$ we analyzed the hydrogen bonding in supercritical water through combined use of NMR spectroscopy and computer simulation. The number of hydrogen bonds per water molecule at supercritical states was estimated quantitatively and the dipole moment of a water molecule was determined at the supercritical states. Once the dipole moment is given at a supercritical state of interest, it is straightforward to construct an effective potential model suitable for simulating water and aqueous solutions at the supercritical state. In a previous paper, the radial and orientational structures of supercritical water at the pure solvent state were examined by employing effective potential models. ${ }^{15}$

In this series of works, we focus on hydration of nonpolar solutes over a wide range of thermodynamic conditions. At an ambient state, a nonpolar solute usually acts as a "structure maker" and its thermodynamics of hydration is dominated by an unfavorable entropy. ${ }^{2,16-46}$ Since the outstanding properties of hydrophobic hydration are closely related to the unique structural characteristics of ambient liquid water, ${ }^{2,16-46}$ the study of hydration of a nonpolar solute in high-temperature water will be useful to assess the extent of uniqueness of super- and subcritical water. We investigate the thermodynamic and structural aspects of hydration of a nonpolar solute in high-temperature water by performing computer simulations over a wide range of density and temperature. In this paper, we elucidate the thermodynamic aspect by examining the chemical potentials and their enthalpic and entropic components of the methane and hard sphere solutes. In an accompanying paper, we focus on the structural aspect to characterize the microscopic solvation structure around a nonpolar solute. ${ }^{47}$

Methane is an organic solute which involves a weakly attractive part in its interaction with water, and the hard sphere particle is a prototypical nonpolar solute which is purely repulsive to the solvent. These nonpolar solutes mix with water when the high-temperature condition is realized and the pressure is not too high. We analyze the hydration thermodynamics of the nonpolar solutes and discuss the thermodynamic origin of their miscibility with high-temperature water. Especially, since the elevated temperature is a nonspecific driving force for mixing any two materials, a careful 
treatment of the temperature effect is made to assess the specificity of high-temperature water.

When the attractive part is present in the solute-solvent interaction, it may make a non-negligible contribution to the hydration thermodynamics. In the scaled-particle theory, the process of inserting a solute into the solvent is decomposed into the formation process of a cavity and the introduction process of the solute to the cavity according to the (soft) solute-solvent interaction..$^{20,21,23-25}$ As emphasized by Pohorille and Pratt, the thermodynamics of cavity formation is closely related to the microscopic inhomogeneity of pure solvent water. ${ }^{39,41,43,46}$ Since the microscopic structure of water changes drastically with a large variation in the thermodynamic condition, it is also of interest to study the thermodynamics of cavity formation over a wide range of density and temperature. The thermodynamics of cavity formation is then related to the size distribution of cavities in the pure solvent, and the connection to the scaled-particle theory is discussed.

In their comprehensive treatment of aqueous solutions of nonpolar solutes, Guillot and Guissani examined the thermodynamics of hydration on the water saturation curve. ${ }^{48}$ When the density and temperature are varied along the saturation curve, a correspondence to experimental studies can be easily made since the solubility data in high-temperature water has often been accumulated on the saturation curve. ${ }^{49-51}$ For the sake of comprehending the general trend of the hydration thermodynamics of a nonpolar solute in high-temperature water, however, it will be insightful to separately treat the density and temperature effects. Furthermore, since the availability of a wide range of density is a most useful characteristic of supercritical water, the effect of density variation is desirable to be studied in supercritical conditions. In this work, we examine the hydration thermodynamics of nonpolar solutes as a function of the density at a fixed supercritical temperature and as a function of the temperature at a fixed liquidlike density.

The thermodynamics of hydration is closely connected to the underlying solvation structure around the solute of interest. In super- and subcritical conditions, it was found, in contrast to the case of ionic hydration, ${ }^{52-63}$ that the hydration is weak around a nonpolar solute. ${ }^{48,52,64,65}$ When the system is close to the critical point, however, the weak perturbation in the solution structure caused by the solute leads to large partial molar thermodynamic quantities of the solute due to extended correlations among solvent molecules. ${ }^{66-72}$ In this case, a simple hydration shell model which accounts for only the contribution from the first hydration shell of the solute will be inadequate to describe the thermodynamics of hydration, and the microscopic structure and energetics of the solution is not straightforwardly inferred from the macroscopic thermodynamic quantities of hydration. In the present paper, we focus only on the thermodynamics of hydration. The relationship between the hydration thermodynamics and the solution structure is pursued in the accompanying paper within the framework of the hydration shell analysis. ${ }^{47,73-75}$

The organization of the present paper is as follows. The simulation methodology is described in Sec. II, and the density fluctuations in pure water at high-temperature states are briefly characterized in Sec. III. The thermodynamics of methane hydration is examined in Sec. IV. The excess chemical potential and the affinity (solubility) of methane for water are described in Sec. IV A, and the decomposition into the enthalpic and entropic components is performed in Sec. IV B. The effect of the (soft) solute-solvent interaction is also pursued in Sec. IV C. In Sec. V, the thermodynamics of cavity formation in high-temperature water is studied and its connection to the scaled-particle theory is discussed.

\section{METHODS}

The thermodynamic quantities of hydration are evaluated from computer simulations of pure solvent water by employing the particle insertion method. ${ }^{76-80}$ The particle insertion method gives the chemical potential of a solute in ambient and high-temperature water and the enthalpy and entropy of hydration are obtained from the temperature dependence of the chemical potential. As far as the system is large enough, the chemical potential is independent of the ensemble and the choice of the ensemble is not important in the particle insertion method. In order to decompose the chemical potential into the enthalpic and entropic components, however, it is necessary to specify the condition of solute insertion, for example, whether the solute is inserted in the constant volume condition or in the constant pressure condition. In previous papers, we showed that the enthalpy and entropy of hydration in the constant pressure condition are connected to the local structure and energetics around the solute with physically transparent variables. ${ }^{73-75}$ Thus, we perform the decomposition of the chemical potential into the enthalpic and entropic components in the constant pressure condition. In order to evaluate the enthalpy and entropy of hydration at constant pressure from the temperature dependence of the chemical potential, it is convenient to vary the temperature under the constant pressure condition. In this work, the computer simulations of pure solvent water to carry out the particle insertion method are implemented in the isothermal-isobaric ensemble.

The ambient to subcritical states of interest are specified by the temperatures of $25,100,200$, and $300{ }^{\circ} \mathrm{C}$ and the pressures corresponding to the water density of $1.0 \mathrm{~g} / \mathrm{cm}^{3}$, which are denoted by the states $A, B, C$, and $D$, respectively. The supercritical states of interest are specified by the temperature of $400{ }^{\circ} \mathrm{C}$ and the pressures corresponding to the water densities of $1.0,0.8,0.6,0.4$, and $0.2 \mathrm{~g} / \mathrm{cm}^{3}$, which are denoted by the states $E, F, G, H$, and $I$, respectively. See Table I for specification. In the thermodynamic path $A \rightarrow B$ $\rightarrow C \rightarrow D \rightarrow E$, the temperature is increased at a fixed density, and in the thermodynamic path $E \rightarrow F \rightarrow G \rightarrow H \rightarrow I$, the density is varied at constant temperature. Our set of the thermodynamic states studied is thus useful to treat the density and temperature effects separately. It is actually parallel to the one employed by Yoshii et al. in their study of supercritical pure water using a polarizable model. ${ }^{81}$

The SPC/E model is adopted as the intermolecular potential function between water molecules. ${ }^{82}$ The critical point of this water model was determined by Guissani and Guillot and was found to be close to the experimental value. ${ }^{83}$ In real water, the dipole moment of a water molecule is a function 
TABLE I. Thermodynamic states of interest.

\begin{tabular}{|c|c|c|c|c|c|}
\hline \multirow[b]{2}{*}{ State } & \multirow[b]{2}{*}{$\begin{array}{c}\text { Temperature } \\
\left({ }^{\circ} \mathrm{C}\right)\end{array}$} & \multicolumn{2}{|c|}{ Canonical ensemble } & \multicolumn{2}{|c|}{ Isothermal-isobaric ensemble } \\
\hline & & $\begin{array}{l}\text { Density }^{\mathrm{a}} \\
\left(\mathrm{g} / \mathrm{cm}^{3}\right)\end{array}$ & $\begin{array}{c}\text { Average pressure } \\
\text { (bar) }\end{array}$ & $\begin{array}{l}\text { Pressure } \\
\text { (bar) }\end{array}$ & $\begin{array}{l}\text { Average density } \\
\qquad\left(\mathrm{g} / \mathrm{cm}^{3}\right)\end{array}$ \\
\hline$A$ & 25 & & & 1 & 1.00 \\
\hline$B$ & 100 & 1.00 & $993 \pm 15$ & 993 & 1.00 \\
\hline$C$ & 200 & 1.00 & $2949 \pm 20$ & 2949 & 1.00 \\
\hline$D$ & 300 & 1.00 & $5109 \pm 12$ & 5109 & 1.00 \\
\hline E & 400 & 1.00 & $7332 \pm 11$ & 7332 & 1.00 \\
\hline$F$ & 400 & 0.80 & $2303 \pm 7$ & 2303 & 0.80 \\
\hline$G$ & 400 & 0.60 & $663 \pm 2$ & 663 & 0.60 \\
\hline$H$ & 400 & 0.40 & $307 \pm 2$ & 307 & 0.39 \\
\hline$I$ & 400 & 0.20 & $225 \pm 1$ & 225 & 0.20 \\
\hline
\end{tabular}

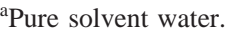

${ }^{\mathrm{b}}$ The values are evaluated in pure solvent water.

${ }^{c}$ The values are evaluated in pure solvent water. The errors are less than $0.01 \mathrm{~g} / \mathrm{cm}^{3}$.

of the density and temperature of the system. When the density is lower and/or the temperature is higher, the dipole moment of a water molecule is smaller and the attractive interaction between water molecules is weaker. In the SPC/E model, however, the state-dependent nature of the dipole moment is not incorporated. The state dependence of the dipole moment can be implemented by employing a polarizable model or an effective potential model with a modified dipole moment. ${ }^{15,81,84-95}$ In the present work, we adopt the SPC/E model over all the states of interest. This is justified because the objective of the present work is to elucidate the general trend of the hydration thermodynamics of a nonpolar solute in high-temperature water. Actually, it was estimated in Ref. 15 that even at the low- to medium-density states of the supercritical region (the states $G, H$, and $I$ in Table I), the value of the dipole moment is closer to that in the SPC/E model than to that in the dilute gas phase. The effect of the reduced dipole moment at supercritical states on the hydration thermodynamics is treated in Appendix A.

The input pressure needs to be specified to perform a simulation in the isothermal-isobaric ensemble. At the state $A$ in Table $\mathrm{I}$, the input pressure is clearly 1 bar. To determine the input pressures at the states other than $A$, we carried out Monte Carlo statistical-mechanical simulations of pure solvent water in the canonical ensemble with the input densities and temperatures shown in Table I. In each Monte Carlo simulation, 648 water molecules were located in a cubic unit cell and the standard Metropolis sampling scheme was implemented. ${ }^{76,96}$ The Monte Carlo simulation was performed for one million passes, where one pass corresponds to the generation of 648 configurations. The intermolecular interactions between water molecules were spherically truncated at $9.0 \AA$, and the periodic boundary condition in the minimum image convention was employed. The average pressures at the states other than $A$ are shown in Table I. These average pressures are used as the input pressures of the simulations performed in the isothermal-isobaric ensemble. In Table I, the pressure and temperature are specified for each thermodynamic state of interest. For each state of interest, two additional states were simulated to evaluate the temperature derivative of the chemical potential under the constant pressure condition. The state of interest and the cor- responding additional states involve the same pressure but different temperatures. At the state $A$ in Table I, the temperatures of the additional states were taken to be 5 and $45^{\circ} \mathrm{C}$. Similarly, for each of the states $B, C, D, E, F$, and $G$, the differences between its own temperature and the temperatures of the additional states were $\pm 20^{\circ} \mathrm{C}$. At the states $H$ and $I$, the temperature differences were set to $\pm 10^{\circ} \mathrm{C}$ since these states are not far from the critical point and involve large thermal expansion coefficients. At each of the states in Table I and the additional states, a Monte Carlo simulation of the pure water system was carried out in the isothermalisobaric ensemble for one million passes by locating 648 water molecules in a cubic unit cell. ${ }^{76,96}$ The truncation scheme for the intermolecular interactions and the boundary condition were identical to those described above for the simulations of pure water in the canonical ensemble. Table I shows that the average densities in the isothermal-isobaric ensemble are coincident with the input densities of the corresponding simulations in the canonical ensemble, as expected. In the following, we specify the state of a system simulated in the isothermal-isobaric ensemble by the temperature and the average water density, rather than by the temperature and the input pressure.

In this work, the hydration thermodynamics of a nonpolar solute is analyzed in terms of the excess chemical potential and its derivatives at infinite dilution. The excess chemical potential $\Delta \mu$ of a solute is the change in the free energy of the system when the solute is inserted at a fixed position in the fluid. In other words, $\Delta \mu$ is equal to the deviation of the (total) chemical potential of the solute in the fluid of interest from that in the ideal gas involving the same density and temperature as the fluid. ${ }^{17,18} \mathrm{~A}$ derivative of the excess chemical potential is called an excess partial molar thermodynamic quantity. For example, the excess partial molar enthalpy and entropy in the constant pressure condition, which are denoted by $\Delta H_{P}$ and $\Delta S_{P}$, respectively, are connected to $\Delta \mu$ through

$$
\Delta H_{P}=-T^{2}\left(\frac{\partial(\Delta \mu / T)}{\partial T}\right)_{P}, \quad \Delta S_{P}=-\left(\frac{\partial \Delta \mu}{\partial T}\right)_{P},
$$

where $T$ is the temperature and $P$ is the pressure. It should be 
noted that while $\Delta \mu$ does not depend on the condition of solute insertion, its derivatives do in general. An excess partial molar thermodynamic quantity is particularly useful for analyzing the solution structure around the solute since it does not involve the ideal (translational) contribution and is equal to the change in the corresponding thermodynamic quantity of the total system upon insertion of the solute at a fixed position in the fluid. ${ }^{17,18,73-75}$

When the solute is distributed in equilibrium between the solution phase of interest and the gas phase, its solubility is commonly represented by the Ostwald coefficient $\gamma$, the ratio of the solute density in the solution phase to that in the gas phase. It is well known that $\gamma$ is determined by the ratio of the difference between the excess chemical potentials of the solute in the solution phase and in the gas phase to the thermal energy (the product of the Boltzmann constant $k_{B}$ and the temperature $T){ }^{17,18}$ In particular, when the solute concentration is low and the gas phase may be treated as an ideal gas, $\gamma$ is related to the excess chemical potential $\Delta \mu$ in the solution at infinite dilution through

$$
\gamma=\exp (-\beta \Delta \mu)
$$

where $\beta$ is the inverse of the thermal energy. Equation (2) shows that a smaller $\beta \Delta \mu$ is equivalent to an enhanced solubility (a larger Ostwald coefficient). ${ }^{97}$ When the concentration of the solute is not low enough or the gas phase is imperfect, Eq. (2) does not hold and $\Delta \mu$ is not directly related to the solubility of the solute. Even in this case, $\Delta \mu$ is the free energy penalty of inserting the solute molecule into the solvent at infinite dilution and $\beta \Delta \mu$ serves as a measure of the affinity of the nonpolar solute for water. Indeed, $\exp (-\beta \Delta \mu)$ is equal to the Ostwald coefficient between the dilute solution phase of interest and the hypothetical ideal gas phase and is called the solubility parameter by Guillot and Guissani. ${ }^{48}$ In order to see the importance of the form $\beta \Delta \mu$, it is insightful to consider the hypothetical equilibrium of the solute distribution between two aqueous phases involving different sets of water density and temperature. In this hypothetical system, the two phases are thermally and mechanically insulated from each other and only the transfer of the solute molecules is allowed between the phases. When the concentration of the solute is low, the equilibrium ratio of the solute densities in the two phases is given by $\exp (-\delta(\beta \Delta \mu))$, where $\delta(\beta \Delta \mu)$ is equal to the difference between $\beta \Delta \mu$ in the two phases. The solute density is then higher in the phase with the smaller $\beta \Delta \mu$. In other words, the affinity of the solute for the solvent is higher when $\beta \Delta \mu$ is smaller. In this paper, we represent the affinity of a nonpolar solute for water by $\beta \Delta \mu$ (or its monotonic function). The affinity is enhanced when $\beta \Delta \mu$ is decreased, and it is reduced when $\beta \Delta \mu$ is increased. Of course, the affinity has the same meaning as the solubility (the Ostwald coefficient) when Eq. (2) holds.

The nonpolar solutes treated in this work are the methane and hard sphere molecules. These nonpolar solutes were treated as test particles and the particle insertion method was employed to calculate their excess chemical potentials at infinite dilution. ${ }^{76-80}$ The methane-water interaction was taken to be the Lennard-Jones interaction with
$\epsilon_{\mathrm{Me}-\mathrm{O}}=0.21 \mathrm{kcal} / \mathrm{mol}$ and $\sigma_{\mathrm{Me}-\mathrm{O}}=3.45 \AA{ }^{98}$ and was also spherically truncated at $9.0 \AA$. The methane-water interaction employed in the present work is different from the one employed by Guillot and Guissani in that the effect of the solute polarizability is not included in our methane-water interaction. ${ }^{48}$ Our simplified choice of the solute-solvent interaction will be justified because the aim of this work is to comprehend the general trend of the thermodynamic behavior of hydration of a nonpolar solute over a wide range of density and temperature, rather than to accurately reproduce experimentally observable thermodynamic quantities. When the particle insertion method is employed to calculate the excess chemical potential of a hard sphere solute of exclusion radius $\lambda$, the successful insertion of the solute means that the distance between the center of the inserted hard sphere and the oxygen site of any water molecule is at least $\lambda \cdot{ }^{23-25,39,41,43}$ When the probability of successful insertion of the hard sphere of exclusion radius $\lambda$ is $p(\lambda)$, the excess chemical potential $\Delta \mu$ of the hard sphere solute is given by ${ }^{99}$

$$
\Delta \mu=-k_{B} T \ln p(\lambda) \text {. }
$$

For both the methane and hard sphere solutes, the excess partial molar enthalpy and entropy at constant pressure were evaluated through finite difference by employing Eq. (1).

The particle insertion method is a highly efficient method to evaluate the excess chemical potential of a solute when the rate of successful insertion of the solute is not too low. ${ }^{76-80}$ Beutler et al. examined the validity of the particle insertion method for purely repulsive solutes in ambient SPC water ${ }^{100}$ and reported that the method is not very accurate when the thermal radius of the solute-solvent repulsive core exceeds $3 \AA .{ }^{79}$ On the other hand, Smith showed that up to the core radius of $4 \AA$, the particle insertion method is a valid procedure to calculate the excess chemical potential in ambient SPC and SPC/E water when a large number of insertions are attempted ${ }^{80}$ In Appendix B, we compare the particle insertion method and the thermodynamic integration method for our systems of interest and demonstrate the validity of the particle insertion method.

In order to evaluate the interaction of a nonpolar solute with the solvent water, Monte Carlo simulations of aqueous solution of methane were also performed in the isothermalisobaric ensemble at the states listed in Table I. In these simulations, 648 water molecules and 1 methane molecule were located in a cubic unit cell, and the position of the solute was fixed at the center of the unit cell. The interaction potentials and the boundary condition were the same as those for the above simulations of pure water implementing the particle insertion method. At each state in Table I, the simulation was performed for one million passes.

\section{STRUCTURE OF PURE SOLVENT WATER}

The purpose of this paper is to elucidate the thermodynamics of hydration of a nonpolar solute in high-temperature water. Since the hydration thermodynamics is closely related to the structure and fluctuation of pure solvent water, it is useful to examine the local and global inhomogeneities in high-temperature water at the outset. In this section, we show 

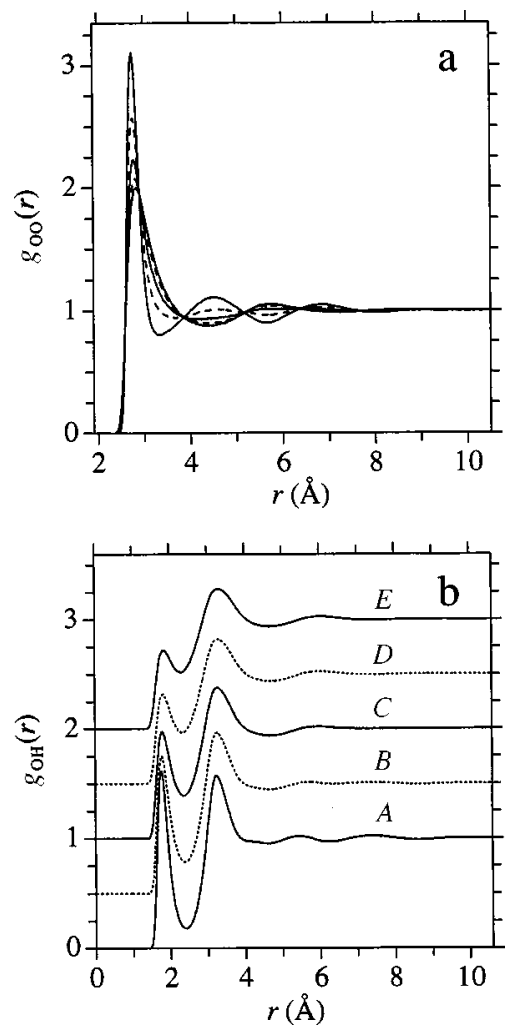

FIG. 1. (a) The O-O radial distribution functions $g_{\mathrm{OO}}$ at the states $A, B, C$, $D$, and $E$, which involve densities of $1.0 \mathrm{~g} / \mathrm{cm}^{3}$. In the region $r \leqslant 3.9 \AA, g_{\text {OO }}$ at a lower temperature is sharper. In the region $3.9 \AA \lessgtr r \lesssim 5.2 \AA, g_{\text {OO }}$ is larger at a lower temperature, while in the region $5.2 \AA \lessgtr r \lesssim 6.4 \AA, g_{\text {OO }}$ is larger at a higher temperature. The solid lines represent the states $A, C$, and $E$, and the dashed lines represent the states $B$ and $D$. (b) The O-H radial distribution functions $g_{\mathrm{OH}}$ at the states $A, B, C, D$, and $E$. The solid lines represent the states $A, C$, and $E$, and the dotted lines represent the states $B$ and $D . g_{\mathrm{OH}}$ is shifted by $0.5,1.0,1.5$, and 2.0 at the states $B, C, D$, and $E$, respectively.

the radial distribution functions, the isothermal compressibility, and the thermal expansion coefficient of ambient and high-temperature water and describe their characteristics briefly.

The microscopic structure of water is typically described in terms of the correlation functions between the intermolecular pairs of atoms $\mathrm{O}-\mathrm{O}, \mathrm{O}-\mathrm{H}$, and $\mathrm{H}-\mathrm{H}$. In particular, the hydrogen bond network involving three or more water molecules is characterized by the $\mathrm{O}-\mathrm{O}$ radial distribution function $\left(g_{\mathrm{OO}}\right)$. In Fig. 1(a), we show the effect of temperature variation on $g_{\mathrm{OO}}$ at a fixed density of $1.0 \mathrm{~g} / \mathrm{cm}^{3}$. The response of the first and second peaks of $g_{\mathrm{OO}}$ to the temperature variation is in agreement with that reported for a polarizable model. ${ }^{81}$ The first peak is broader and is positioned at a larger distance as the temperature is raised. In the region where the second peak is present at the ambient state $A$ (3.9 $\AA \lesssim r \lessgtr 5.2 \AA), g_{\text {OO }}$ decreases monotonically with the temperature and involves a minimum at temperatures higher than $\sim 200^{\circ} \mathrm{C}$. In the region $5.2 \AA \lessgtr r \lesssim 6.4 \AA$, on the other hand, $g_{\text {OO }}$ increases monotonically with the temperature. When the temperature is higher than $\sim 200^{\circ} \mathrm{C}$, the second peak of $g_{\text {OO }}$ is present at $\sim 6 \AA$, and water behaves like a simple liquid in the sense that the position of the second peak corresponds to twice the distance of the first peak. Further-
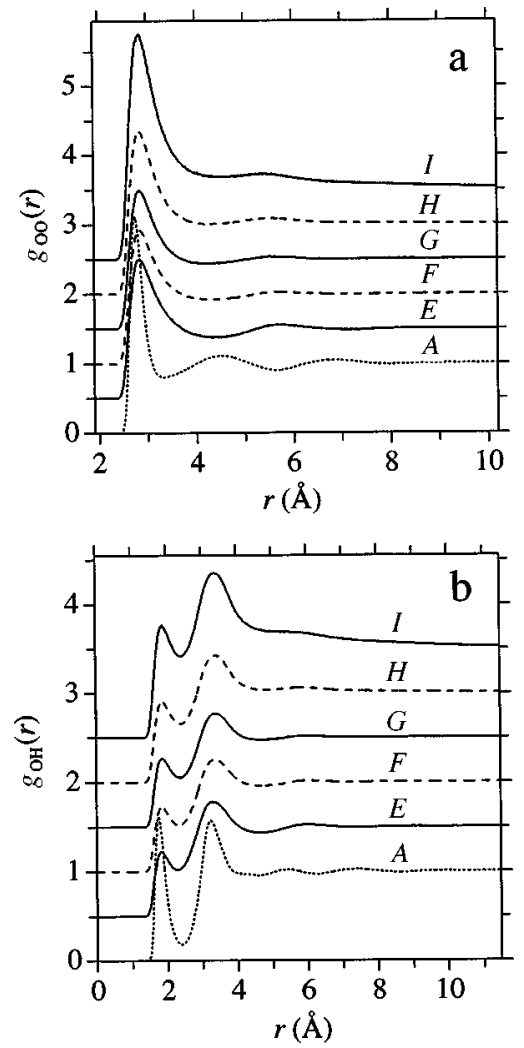

FIG. 2. (a) The $\mathrm{O}-\mathrm{O}$ radial distribution functions $g_{\mathrm{OO}}$ and (b) the $\mathrm{O}-\mathrm{H}$ radial distribution functions $g_{\mathrm{OH}}$ at the ambient state $A$ and at the supercritical states $E, F, G, H$, and $I$. The dotted lines represent the state $A$, the solid lines represent the states $E, G$, and $I$, and the dashed lines represent the states $F$ and $H . g_{\mathrm{OO}}$ and $g_{\mathrm{OH}}$ are shifted by $0.5,1.0,1.5,2.0$, and 2.5 at the states $E, F, G, H$, and $I$, respectively.

more, it was also observed by Guissani and Guillot that the transition to the simple liquid-like behavior occurs between $\sim 150$ and $\sim 200^{\circ} \mathrm{C}$ when the temperature is raised along the liquid branch of the saturation curve. ${ }^{83}$ Note that the change in the second peak of $g_{\text {OO }}$ shown in Fig. 1(a) can be seen as disappearance of one peak at the lower temperatures and emergence of another at the higher temperatures, rather than as a gradual shift in the peak position. The hydrogen bonding between a pair of water molecules is commonly analyzed in terms of the $\mathrm{O}-\mathrm{H}$ radial distribution function $\left(\mathrm{g}_{\mathrm{OH}}\right)$. In Fig. 1(b), we show the temperature dependence of $g_{\mathrm{OH}}$ at a fixed density of $1.0 \mathrm{~g} / \mathrm{cm}^{3}$. In this case, the positional changes in the first and second peaks are relatively small. Instead, the principal change in $g_{\mathrm{OH}}$ is observed for the widths of the peaks. When the temperature is higher, the first and second peaks are less distinct and the hydrogen bonding is less sharply characterized.

When the temperature is above the critical, the density can be continuously varied from the gaslike regime to the liquidlike regime. In Fig. 2(a), we show $g_{\mathrm{OO}}$ at the supercritical states $E, F, G, H$, and $I$ and at the ambient state $A$. It is easy to see, in agreement with previous computer simulations, ${ }^{15,52,53,58,81,83,92-94,101-107}$ that the first peak of $g_{\mathrm{OO}}$ is broader and is positioned at a larger distance when the system is supercritical than when the system is ambient. The second peak of $g_{\mathrm{OO}}$ exhibits a more qualitative change. At 
the ambient state $A$, the second peak is evident at $\sim 4.5 \AA$ and represents the tetrahedral ordering of the hydrogen bond network. At the supercritical states, on the other hand, water behaves like a simple liquid in the sense that the position of the second peak is $\sim 6 \AA$ and corresponds to twice the distance of the first peak. ${ }^{15,52,53,58,81,83,92-94,101-107}$ The density dependence of $g_{\mathrm{OH}}$ is shown in Fig. 2(b) at the supercritical states $E, F, G, H$, and $I$ and at the ambient state $A$. According to Fig. 2(b), although the hydrogen bonding does not vanish in the supercritical conditions, the hydrogen bonding peak of $g_{\mathrm{OH}}$ at $\sim 1.8 \AA$ is less sharply characterized at the supercritical states than at the ambient state. When the density effect is concerned at a fixed supercritical temperature of $400{ }^{\circ} \mathrm{C}$, both $g_{\mathrm{OO}}$ and $g_{\mathrm{OH}}$ decrease with the density over the range of $r$ in Fig. 2 at the low-to medium-density states $G, H$, and $I$. This shows that the association of a specific pair of water molecules is more probable at a lower density, in agreement with the observation from the density dependence of the proton chemical shift that the hydrogen bonding is spatially more inhomogeneous at a lower density. ${ }^{13-15}$ When the density is medium to high $\left(\rho \geq 0.6 \mathrm{~g} / \mathrm{cm}^{3}\right), g_{\mathrm{OO}}$ and $g_{\mathrm{OH}}$ exhibit relatively small changes. In this case, the density dependences of such quantities as the coordination number and the number of hydrogen bonds are governed by the bulk density.

While the local and microscopic fluctuations in the density are described by the radial distribution functions, the global and macroscopic fluctuations in the density are related to the isothermal compressibility $\kappa_{T}$ and the thermal expansion coefficient $\alpha_{P}$ of the system. $\kappa_{T}$ is determined by the fluctuation in the volume (or density) of the system, and $\alpha_{P}$ is a measure of the strength of the coupling between the fluctuations in the density and enthalpy (or energy) of the system. In Fig. 3(a), we show $\kappa_{T}$ as a function of the temperature $T$ at a fixed density of $1.0 \mathrm{~g} / \mathrm{cm}^{3}$. It is seen in agreement with the experimental observations ${ }^{2,108}$ that $\kappa_{T}$ decreases monotonically with $T$. In other words, the system is less compressible at a higher temperature, where the interaction between water molecules is more repulsive and the pressure is higher. In order to relate $\kappa_{T}$ to the magnitude of the fluctuation in the volume (or density), we employ the equation in the isothermal-isobaric ensemble that

$$
\rho k_{B} T \kappa_{T}=1+\rho \int d \mathbf{r}\left(g_{\mathrm{OO}}(r)-1\right)=\rho \frac{\left\langle(\widetilde{V}-\langle\widetilde{V}\rangle)^{2}\right\rangle}{\langle\widetilde{V}\rangle}
$$

where $\rho$ is the density of the system, $\widetilde{V}$ is the instantaneous volume of the system, and $\langle\ldots\rangle$ denotes the ensemble average. ${ }^{76,109}$ The temperature dependence of $\rho k_{B} T \kappa_{T}$ at constant density is shown in Fig. 3(a). According to Fig. 3(a), $\rho k_{B} T \kappa_{T}$ increases with $T$ up to $\sim 300^{\circ} \mathrm{C}$ and the temperature elevation in this region leads to a larger fluctuation in the volume (or density) of the system. At temperatures higher than $\sim 300{ }^{\circ} \mathrm{C}$, on the other hand, the magnitude of the volume fluctuation is weakly dependent on $T$ when seen in terms of the macroscopic quantity $\rho k_{B} T \kappa_{T}$. In Fig. 3(b), we show $\alpha_{P}$ as a function of the temperature $T$ at a fixed density of $1.0 \mathrm{~g} / \mathrm{cm}^{3}$. Unlike $\kappa_{T}, \alpha_{P}$ is a nonmonotonic function of $T$. Actually, $\alpha_{P}$ involves a maximum at
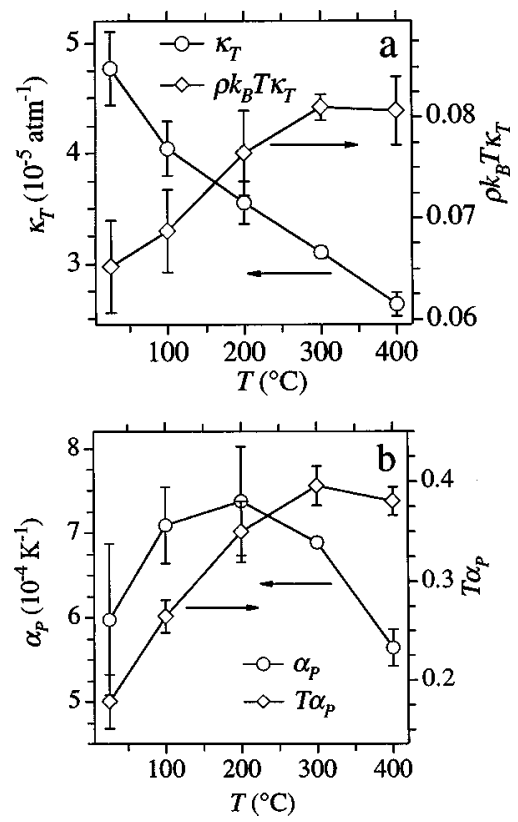

FIG. 3. (a) The isothermal compressibility $\kappa_{T}$ and its normalized form $\rho k_{B} T \kappa_{T}$ as functions of the temperature $T$ at the states $A, B, C, D$, and $E$, which involve densities of $1.0 \mathrm{~g} / \mathrm{cm}^{3}$. (b) The thermal expansion coefficient $\alpha_{P}$ and its normalized form $T \alpha_{P}$ as functions of the temperature $T$ at the states $A, B, C, D$, and $E$. When not shown, the error bar is smaller than the size of the corresponding symbol.

$\sim 200^{\circ} \mathrm{C}$, in agreement with the experimental findings. ${ }^{2,108}$ It is of interest to note, as seen in Fig. 1(a), that the transition to the simple liquid-like behavior is also found for the radial distribution function at $\sim 200^{\circ} \mathrm{C}$. The strength of the coupling between the fluctuations in the volume (or density) and the enthalpy (or energy) of the system is related to $\alpha_{P}$ through the identity in the isothermal-isobaric ensemble that

$$
T \alpha_{P}=\frac{\langle(\widetilde{V}-\langle\widetilde{V}\rangle)(\widetilde{E}+P \widetilde{V}-\langle\widetilde{E}+P \widetilde{V}\rangle)\rangle}{k_{B} T\langle\widetilde{V}\rangle},
$$

where $\widetilde{E}$ is the instantaneous energy and the coupling strength is normalized by the thermal energy $k_{B} T .^{76,109}$ The temperature dependence of $T \alpha_{P}$ at constant density is shown in Fig. 3(b). According to Fig. 3(b), $T \alpha_{P}$ is an increasing function of $T$ up to $\sim 300{ }^{\circ} \mathrm{C}$. In this region, the coupling between the volume and enthalpy of the system is strengthened with the temperature elevation. When the temperature exceeds $\sim 300{ }^{\circ} \mathrm{C}$, on the other hand, $T \alpha_{P}$ is a weak function of $T$. In the framework of the scaled-particle theory, ${ }^{23-25,38,44,46}$ the thermodynamics of cavity formation is more enthalpic when $T \alpha_{P}$ is larger. It is then expected from Fig. 3(b) that the thermodynamics of hydration of a nonpolar solute is enthalpic in the high-temperature regions. In Secs. IV and $\mathrm{V}$, we address this point in detail.

When the density is varied in supercritical conditions, $\kappa_{T}$ and $\alpha_{P}$ change by orders of magnitude. In Fig. 4, we show $\kappa_{T}$ as a function of the density $\rho$ at a fixed temperature of $400{ }^{\circ} \mathrm{C}$. It is indeed seen, in agreement with the experimental observations and the simulation results using the TIP4P model, ${ }^{2,106,108}$ that $\kappa_{T}$ rises rapidly as the density decreases. Actually, the $\rho$ dependence of $\kappa_{T}$ is so strong that $\rho k_{B} T \kappa_{T}$ 


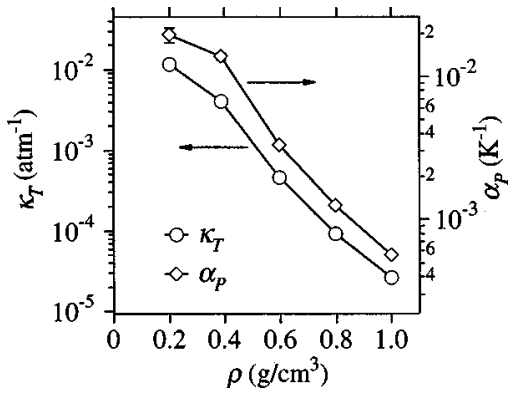

FIG. 4. The isothermal compressibility $\kappa_{T}$ and the thermal expansion coefficient $\alpha_{P}$ as functions of the density $\rho$ at the supercritical states $E, F, G, H$, and $I$. When not shown, the error bar is smaller than the size of the corresponding symbol.

defined by Eq. (4) also increases by orders of magnitude when the density is reduced. Thus, Fig. 4 implies that the volume (or density) fluctuation of the system given by Eq. (4) is smaller at a higher density. The density dependence of $\alpha_{P}$ at constant temperature is also shown in Fig. 4. According to Fig. 4, the density reduction leads to an increase in $\alpha_{P}$ by orders of magnitude and the $\rho$ dependence of $\alpha_{P}$ corresponds qualitatively to that of $\kappa_{T}$. Thus, in the isothermal variation of the density examined in this work, the thermodynamic response functions concerning the density (or volume) fluctuation of the system are larger at a lower density.

\section{THERMODYNAMICS OF METHANE HYDRATION}

In this section, we focus on the thermodynamics of methane hydration. In Sec. IV A, we treat the excess chemical potential and discuss the affinity (solubility) of methane for water over a wide range of density and temperature. In Sec. IV B we perform the decomposition into the enthalpic and entropic components. The thermodynamic characteristics presented in Secs. IV A and IV B for the methane solute are also valid for the hard sphere solutes with the sizes treated in Sec. V. In Sec. IV C we examine the effect of the (soft) solute-solvent interaction on the thermodynamics of methane hydration. We address this issue to clarify the role of the weak solute-solvent attraction at high temperatures.

\section{A. Excess chemical potential}

In Fig. 5(a) we show the excess chemical potential $\Delta \mu$ of methane as a function of the temperature $T$ at a fixed water density of $1.0 \mathrm{~g} / \mathrm{cm}^{3}$. It is evident that $\Delta \mu$ increases with $T$ in the constant volume condition. In other words, the free energy penalty $\Delta \mu$ of inserting the nonpolar solute is larger at a higher temperature, whereas high-temperature water behaves like a simple liquid as seen in Fig. 1(a). ${ }^{110}$ Since the excess partial molar entropy at constant volume $\Delta S_{V}$ is given by

$$
\Delta S_{V}=-\left(\frac{\partial \Delta \mu}{\partial T}\right)_{V},
$$

where $V$ is the volume of the system, Fig. 5(a) shows that $\Delta S_{V}$ is negative over a wide range of temperature shown. In order to understand the affinity of the nonpolar solute for water, it is actually necessary to examine $\beta \Delta \mu$, rather than
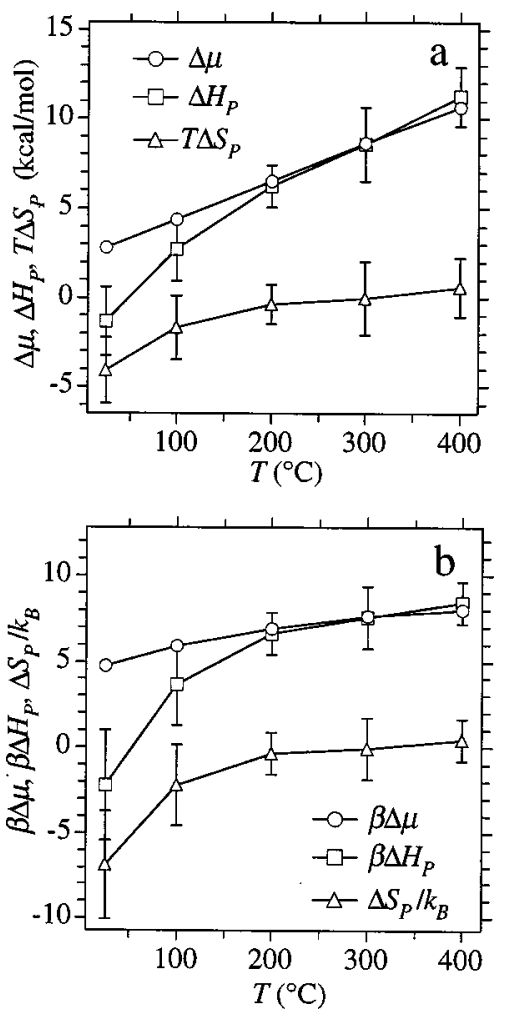

FIG. 5. (a) The excess chemical potential $\Delta \mu$ and its enthalpic and entropic components $\Delta H_{P}$ and $T \Delta S_{P}$ of methane as functions of the temperature $T$ at the states $A, B, C, D$, and $E$, which involve water densities of $1.0 \mathrm{~g} / \mathrm{cm}^{3}$. (b) $\beta \Delta \mu$ and its enthalpic and entropic components $\beta \Delta H_{P}$ and $\Delta S_{P} / k_{B}$ of methane as functions of the temperature $T$ at the states $A, B, C, D$, and $E$. When not shown, the error bar is smaller than the size of the corresponding symbol.

$\Delta \mu$ itself, as described in Sec. II. In Fig. 5(b), we show $\beta \Delta \mu$ as a function of $T$ at constant density. Although $\beta \Delta \mu$ exhibits a weaker temperature dependence than $\Delta \mu$ due to the factor $\beta$, it is still an increasing function of the temperature. Thus, over a wide temperature range shown in Fig. 5, the affinity (solubility) of the nonpolar solute decreases with $T$. In this sense, methane is more "hydrophobic" at higher temperatures when the density of water is fixed, whereas the uniqueness of the water structure is diminished by elevating the temperature as shown in Fig. 1. It may then be misleading to ascribe the origin of hydrophobicity only to the unique structural characteristic of water, such as the tetrahedral ordering. Since the excess partial molar energy at constant volume $\Delta E_{V}$ is expressed as

$$
\Delta E_{V}=-T^{2}\left(\frac{\partial(\Delta \mu / T)}{\partial T}\right)_{V},
$$

Fig. 5(b) also implies that $\Delta E_{V}$ is negative at ambient to supercritical temperatures. Detailed analyses of the energetic (enthalpic) and entropic components are presented in Sec. IV B.

When the temperature is raised above the critical, there is no prohibited region between the gas-like and liquid-like densities. In Fig. 6(a), we show the excess chemical potential $\Delta \mu$ of methane as a function of the water density $\rho$ at a fixed temperature of $400{ }^{\circ} \mathrm{C}$, along with the reference value at the 

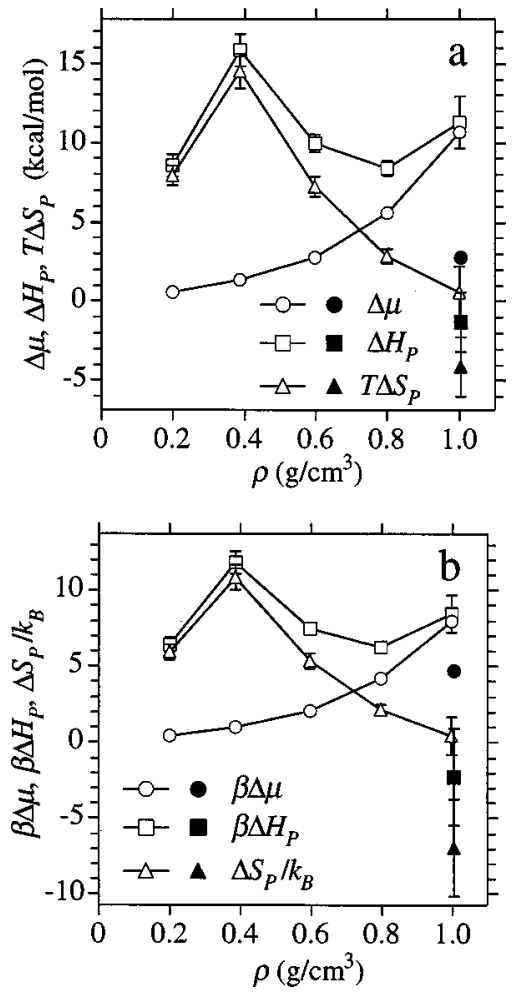

FIG. 6. (a) The excess chemical potential $\Delta \mu$ and its enthalpic and entropic components $\Delta H_{P}$ and $T \Delta S_{P}$ of methane as functions of the density $\rho$ at the ambient state $A$ and at the supercritical states $E, F, G, H$, and $I$. (b) $\beta \Delta \mu$ and its enthalpic and entropic components $\beta \Delta H_{P}$ and $\Delta S_{P} / k_{B}$ of methane as functions of the density $\rho$ at the ambient state $A$ and at the supercritical states $E, F, G, H$, and $I$. The filled symbols represent the state $A$ and the open symbols connected by solid lines represent the states $E, F, G, H$, and $I$. When not shown, the error bar is smaller than the size of the corresponding symbol.

ambient state (the state $A$ in Table I). It is evident that $\Delta \mu$ increases with $\rho$ in the constant temperature condition and that the excess partial molar volume is positive in the density range shown in Fig. 6(a). In the low-density region ( $\rho$ $\lesssim 0.4 \mathrm{~g} / \mathrm{cm}^{3}$ ) of the supercritical states, $\Delta \mu$ is smaller than that at the ambient state $A$. This simply reflects the fact that $\Delta \mu$ approaches zero for any fluid in the limit of zero density. In the medium- to high-density region $\left(\rho \geqslant 0.6 \mathrm{~g} / \mathrm{cm}^{3}\right)$ of the supercritical states, on the other hand, $\Delta \mu$ is larger than that at the ambient state $A$. In other words, when seen in terms of the free energy penalty $\Delta \mu$, the insertion of methane is less favorable in supercritical water with $\rho$ $\geq 0.6 \mathrm{~g} / \mathrm{cm}^{3}$ than in ambient water with $\rho=1.0 \mathrm{~g} / \mathrm{cm}^{3}$. ${ }^{110}$ In order to examine the affinity of the nonpolar solute for supercritical water, in Fig. 6(b) we show $\beta \Delta \mu$ as a function of $\rho$ at constant temperature, along with the reference value at the ambient state. Since the (absolute) temperatures at the supercritical states of interest are more than twice the temperature at the ambient state, $\beta \Delta \mu$ becomes smaller at the supercritical states $F\left(\rho=0.8 \mathrm{~g} / \mathrm{cm}^{3}\right)$ and $G(\rho=0.6$ $\mathrm{g} / \mathrm{cm}^{3}$ ) than at the ambient state $A$ when the factor $\beta$ is taken into account. This shows that in the medium-density region $\left(0.6 \mathrm{~g} / \mathrm{cm}^{3} \leq \rho \leq 0.8 \mathrm{~g} / \mathrm{cm}^{3}\right)$, the enhanced affinity of the nonpolar solute for supercritical water results from the elevated temperature, not from the free energy penalty.
When the density decreases and/or the temperature increases, water is considered to become less unique as the hydrogen bonding is disrupted. ${ }^{13-15}$ A clear indication of this point is the reduction in the dielectric constant, which is observed in both experimental studies ${ }^{2,108}$ and model calculations using the SPC/E model. ${ }^{111,112}$ Since the strong hydrogen bonding and the large dielectric constant are major unique characteristics of ambient water, water is viewed as more "nonpolar" at lower densities and/or higher temperatures. In solution chemistry, it is a rule of thumb that a nonpolar solute dissolves well into a nonpolar solvent since the dissolution involves a small or no free energy penalty. Figures 5 and 6 show, however, that the free energy penalty $\Delta \mu$ of inserting methane in water is larger at the hightemperature states with the medium to high density ( $\rho$ $\geq 0.6 \mathrm{~g} / \mathrm{cm}^{3}$ ) than at the ambient state. In other words, while high-temperature water is more "oil-like" than ambient water from the viewpoint of the reduced hydrogen bonding and dielectric constant, the increased "oil-likeness" of hightemperature water does not lead to a small free energy penalty $\Delta \mu$. In this case, the enhanced affinity of the nonpolar solute for high-temperature water with the medium density $\left(0.6 \mathrm{~g} / \mathrm{cm}^{3} \leq \rho \leq 0.8 \mathrm{~g} / \mathrm{cm}^{3}\right)$ is simply the effect of the elevated temperature to reduce $\beta \Delta \mu$. The effect of temperature elevation to mix two compounds with an unfavorable $\Delta \mu$ is generally observed and is not specific to water. Therefore, the enhanced affinity (solubility) of the nonpolar solute for super- and subcritical water represents the nonspecificity of super- and subcritical water, rather than the uniqueness, when seen in terms of the free energy penalty of the hydration process.

In their comprehensive treatment of the affinities of nonpolar solutes for SPC/E water along the liquid branch of the water saturation curve, Guillot and Guissani observed, in agreement with the experimental findings, ${ }^{49-51}$ that the affinities involve minima in the temperature variation. ${ }^{48}$ In the present work, it is seen in Figs. 5 and 6 that the affinity of methane decreases when the temperature is raised at constant density and that it increases when the density is reduced at constant temperature. When the temperature is raised along the liquid branch of the saturation curve, the density of water decreases. Thus, the presence of the affinity minima between $\sim 25$ and $\sim 100^{\circ} \mathrm{C}$ on the saturation curve indicates that in the low-temperature regime of the saturation curve, the effect of elevating the temperature to reduce the affinity overwhelms the effect of decreasing the density to enhance the affinity.

\section{B. Excess partial molar enthalpy and entropy}

When the hydration of a nonpolar solute is concerned in ambient conditions, the thermodynamic characterization of the enthalpic and entropic components reveals the unique features of the hydration clearly. It is indeed well known in hydrophobic hydration that while the excess partial molar enthalpy is negative and favorable, the entropic component overwhelms the enthalpic component and gives rise to the low solubility of a nonpolar solute in ambient water. ${ }^{2,16-46}$ When the hydration of a nonpolar solute is concerned in high-temperature conditions, it will also be insightful to ex- 
amine the enthalpic and entropic components of the hydration thermodynamics. In this regard, it should be noted that the excess partial molar enthalpy and entropy are dependent upon whether the solute is inserted in the constant volume condition or in the constant pressure condition. Actually, the excess partial molar energy and entropy in the constant volume condition can be estimated from the temperature dependence of the excess chemical potential shown in Fig. 5. Within the framework of the hydration shell analysis, however, the partial molar quantities in the constant pressure condition have transparent connections with the local structure and energetics around the solute. ${ }^{73-75}$ As noted in Sec. II, in this work the excess partial molar enthalpy $\Delta H_{P}$ and the excess partial molar entropy $\Delta S_{P}$ are evaluated under the constant pressure condition. The hydration shell analysis relating the excess partial molar quantities to the microscopic solvation structure is presented in the accompanying paper. ${ }^{47}$

In Fig. 5(a), we show $\Delta H_{P}$ and $T \Delta S_{P}$ as functions of the temperature $T$ at a fixed water density of $1.0 \mathrm{~g} / \mathrm{cm}^{3}$. In the ambient condition (the state $A$ in Table I), $T \Delta S_{P}$ is unfavorable (negative) and larger in magnitude than $\Delta H_{P}$. This indeed represents the thermodynamic characterization of hydrophobic hydration. As the temperature increases, $T \Delta S_{P}$ becomes smaller in magnitude and $\Delta H_{P}$ is closer to the excess chemical potential $\Delta \mu$. In other words, at higher temperatures, the entropic contribution to $\Delta \mu$ is smaller and the thermodynamics of methane hydration is more enthalpic. Actually, $\Delta \mu$ is essentially enthalpic at temperatures higher than $\sim 200^{\circ} \mathrm{C}$, where Fig. 1(a) shows that pure water exhibits the simple liquid-like behavior. When a spherical cavity is introduced into an organic solvent, it is commonly seen that the free energy of cavity formation is enthalpic. ${ }^{23,25,38,44,46} \mathrm{In}$ this sense, the hydration of the nonpolar solute in the hightemperature region is more of "organic" character than of "aqueous" character since the solute-solvent attractive interaction is shown in Sec. IV C to make a minor contribution to the hydration thermodynamics in the high-temperature region. It should be noted, however, that the increased "organic" character of high-temperature hydration does not assure the enhanced affinity of the nonpolar solute for water, as evidenced from the excess chemical potential in Fig. 5.

When the affinity (solubility) of the solute is to be compared at different temperatures, $\beta \Delta \mu$, rather than $\Delta \mu$ itself, needs to be treated explicitly. Correspondingly, it is necessary to treat $\beta \Delta H_{P}$ and $\Delta S_{P} / k_{B}$ explicitly in order to compare the enthalpic and entropic components of the hydration thermodynamics at different temperatures. In Fig. 5(b), we show $\beta \Delta H_{P}$ and $\Delta S_{P} / k_{B}$ as functions of the temperature $T$ at constant density. It is easy to see that the factor $\beta$ does not alter the general trend of the temperature dependence of the enthalpic and entropic components. To examine the effects of $\Delta H_{P}$ and $\Delta S_{P}$ on the affinity difference between the ambient and high-temperature states, it is insightful to simply decompose the difference of $\beta \Delta \mu$ into the enthalpic and entropic components. Let $\beta^{A}, \Delta \mu^{A}, \Delta H_{P}^{A}$, and $\Delta S_{P}^{A}$ be the $\beta, \Delta \mu, \Delta H_{P}$, and $\Delta S_{P}$ at the ambient state $A$, respectively, and let $\beta^{\mathrm{HT}}, \Delta \mu^{\mathrm{HT}}, \Delta H_{P}^{\mathrm{HT}}$, and $\Delta S_{P}^{\mathrm{HT}}$ be the $\beta, \Delta \mu, \Delta H_{P}$, and $\Delta S_{P}$ at a high-temperature state (the state $B, C, D$, or $E$ ) shown in Fig. 5, respectively. It is then a thermodynamic identity that

$$
\begin{aligned}
\beta^{\mathrm{HT}} \Delta \mu^{\mathrm{HT}}-\beta^{A} \Delta \mu^{A}= & \left(\beta^{\mathrm{HT}} \Delta H_{P}^{\mathrm{HT}}-\beta^{A} \Delta H_{P}^{A}\right) \\
& -\left(\Delta S_{P}^{\mathrm{HT}}-\Delta S_{P}^{A}\right) / k_{B} .
\end{aligned}
$$

According to Eq. (8) and Fig. 5(b), the decrease in the affinity at the high-temperature state expressed as $\beta^{\mathrm{HT}} \Delta \mu^{\mathrm{HT}}$ $>\beta^{A} \Delta \mu^{A}$ is due to $\beta^{\mathrm{HT}} \Delta H_{P}^{\mathrm{HT}}>\beta^{A} \Delta H_{P}^{A}$. In other words, the reduced affinity in the high-temperature region results from the unfavorable change in the enthalpic component upon the temperature elevation. The change in the entropic component stabilizes the nonpolar solute in water at the hightemperature state, but is not large enough to negate the unfavorable change in the enthalpic component. Thus, in view of the decomposition into the enthalpic and entropic components, the affinity change of the nonpolar solute against the temperature variation at constant density is governed by the enthalpic component.

We now perform the enthalpy-entropy decomposition of the hydration thermodynamics by varying the density in the supercritical conditions. In Fig. 6(a), we show $\Delta H_{P}$ and $T \Delta S_{P}$ as functions of the water density $\rho$ at a temperature of $400{ }^{\circ} \mathrm{C}$, along with the reference values at the ambient state (the state $A$ in Table I). It is evident that the excess partial molar enthalpy is favorable (negative) at the ambient state and unfavorable (positive) at the supercritical states, while the excess partial molar entropy is unfavorable (negative) at the ambient state and favorable (positive) at the supercritical states. Furthermore, at the supercritical states, $\Delta H_{P}$ dominates over $T \Delta S_{P}$ and gives rise to the positive excess chemical potential $\Delta \mu$. Unlike the case of $\Delta \mu$, the density dependences of $\Delta H_{P}$ and $\Delta S_{P}$ are not monotonic. There are maxima in both $\Delta H_{P}$ and $\Delta S_{P}$ at $\rho \approx 0.4 \mathrm{~g} / \mathrm{cm}^{3}$ (the state $H)$. The presence of the maxima reflects the fact that $\Delta H_{P}$ and $\Delta S_{P}$ become large near the critical point due to the divergent behavior of the isothermal compressibility of the pure solvent. ${ }^{66-72}$ In the medium- to high-density region ( $\rho$ $\left.\gtrsim 0.6 \mathrm{~g} / \mathrm{cm}^{3}\right), \Delta S_{P}$ decreases with $\rho$, and the hydration thermodynamics is more strongly governed by the enthalpic component at a higher density.

In order to compare the enthalpic and entropic contributions to the hydration thermodynamics at the ambient and supercritical temperatures, in Fig. 6(b) we show $\beta \Delta H_{P}$ and $\Delta S_{P} / k_{B}$ as functions of the water density $\rho$ at a temperature of $400{ }^{\circ} \mathrm{C}$, along with the reference values at the ambient state $A$. The comparison between the ambient state (the state $A$ ) and a supercritical state (the state $E, F, G, H$, or $I$ ) is then made by employing Eq. (8), where the supercritical state is taken as the high-temperature state. Equation (8) and Fig. 6(b) show that in the low- to medium-density region ( $\rho$ $\lesssim 0.8 \mathrm{~g} / \mathrm{cm}^{3}$ ), the increase in the affinity at the supercritical state expressed as $\beta^{\mathrm{HT}} \Delta \mu^{\mathrm{HT}}<\beta^{A} \Delta \mu^{A}$ results from $\Delta S_{P}^{\mathrm{HT}}$ $>\Delta S_{P}^{A}$. In other words, the enhanced affinity at the supercritical state with $\rho \leqq 0.8 \mathrm{~g} / \mathrm{cm}^{3}$ is caused by the entropy gain obtained when the system is moved from the ambient state to the supercritical state. This entropy gain is large enough to negate the unfavorable $\Delta S_{P}$ at the ambient state $A$, which is a thermodynamic signature of hydrophobic hydra- 
tion. The role of the enthalpic component is to partially compensate the entropy gain. Therefore, in view of the decomposition into the enthalpic and entropic components, the affinity change of the nonpolar solute from the ambient state to the supercritical state with the low to medium density ( $\rho$ $\lesssim 0.8 \mathrm{~g} / \mathrm{cm}^{3}$ ) is governed by the entropic component.

When the temperature is raised on the liquid branch of the water saturation curve, it is expected from Figs. 5 and 6 that $\Delta S_{P}$ increases with the temperature. This expectation is indeed supported by the observations of Guillot and Guissani concerning the temperature dependence of $\Delta S_{P}$ on the saturation curve. ${ }^{48}$ Furthermore, Guillot and Guissani showed that $\Delta H_{P}$ also increases with the temperature along the liquid branch of the saturation curve. ${ }^{48}$ This indicates from Figs. 5 and 6 that when the temperature is raised on the saturation curve, the effect of the temperature elevation to increase $\Delta H_{P}$ overwhelms the effect of the density reduction to decrease $\Delta H_{P}$ in the high-density region $\left(\rho \gtrsim 0.8 \mathrm{~g} / \mathrm{cm}^{3}\right)$.

\section{Effect of the solute-solvent interaction}

A realistic solute-solvent interaction usually involves an attractive part even when the solute is sparsely soluble in the solvent. In this case, although the attractive part in the solute-solvent interaction will not play a dominant role in determining the thermodynamic behavior of hydration, it may make a non-negligible contribution to the hydration thermodynamics. A convenient conceptual framework to treat the (soft) solute-solvent interaction has been provided by Pierotti in his developments of the scaled-particle theory. ${ }^{20,21,23-25}$ In Pierotti's developments, the process of the solute insertion is decomposed into two processes. One is the process of forming a cavity to accommodate the solute, and the other is the process of introducing the (soft) solutesolvent interaction. The purpose of this section is to elucidate the role of the (soft) solute-solvent interaction in the hydration thermodynamics. We decompose the thermodynamics of methane hydration into two components in a manner analogous to that given by Pierotti and isolate the effect of the solute-solvent attraction on the affinity of methane for water.

Let $\left\langle u_{\mathrm{uv}}\right\rangle$ be the average sum of the interaction of methane with all the water molecules. We treat the excess chemical potential $\Delta \mu$ of methane simply as a sum of $\left\langle u_{\mathrm{uv}}\right\rangle$ and $\left(\Delta \mu-\left\langle u_{\mathrm{uv}}\right\rangle\right)$, which are called the interaction component and the cavity component, respectively. ${ }^{113-116}$ In Figs. 7(a) and $7(\mathrm{~b})$, we show $\left\langle u_{\mathrm{uv}}\right\rangle$ and $\left(\Delta \mu-\left\langle u_{\mathrm{uv}}\right\rangle\right)$, respectively, as functions of the temperature $T$ at a fixed water density of $1.0 \mathrm{~g} / \mathrm{cm}^{3}$. It is evident that the interaction component is less attractive (less negative) at a higher temperature. The cavity component $\left(\Delta \mu-\left\langle u_{\mathrm{uv}}\right\rangle\right)$ is an increasing function of $T$ and governs the excess chemical potential of methane more strongly at a higher temperature. In addition, Figs. 5(a) and 7 (a) show that $\left\langle u_{\text {uv }}\right\rangle$ plays a minor role in determining the excess partial molar enthalpy $\Delta H_{P}$ at temperatures higher than $\sim 200{ }^{\circ} \mathrm{C}$. In other words, $\Delta H_{P}$ in hightemperature water is dominated by the solvent reorganization term, which describes the shift in the solvent binding energy induced by insertion of the solute. ${ }^{73}$
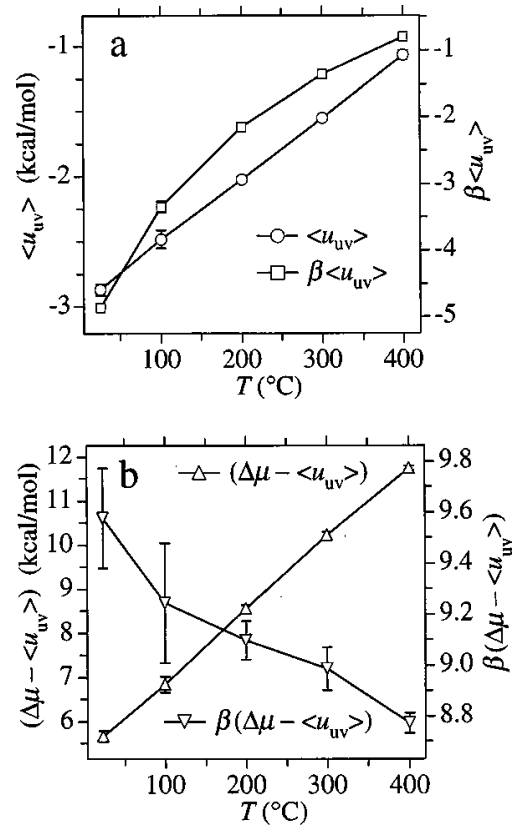

FIG. 7. (a) The average solute-solvent interaction $\left\langle u_{\mathrm{uv}}\right\rangle$ and its normalized form $\beta\left\langle u_{\mathrm{uv}}\right\rangle$ of the hydration thermodynamics of methane as functions of the temperature $T$ at the states $A, B, C, D$, and $E$, which involve water densities of $1.0 \mathrm{~g} / \mathrm{cm}^{3}$. (b) The cavity component $\left(\Delta \mu-\left\langle u_{\mathrm{uv}}\right\rangle\right)$ and its normalized form $\beta\left(\Delta \mu-\left\langle u_{\mathrm{uv}}\right\rangle\right)$ of the hydration thermodynamics of methane as functions of the temperature $T$ at the states $A, B, C, D$, and $E$. When not shown, the error bar is smaller than the size of the corresponding symbol.

In order to see the effects of the interaction and cavity components on the affinity (solubility) of methane, we show $\beta\left\langle u_{\mathrm{uv}}\right\rangle$ and $\beta\left(\Delta \mu-\left\langle u_{\mathrm{uv}}\right\rangle\right)$ in Figs. 7(a) and 7(b), respectively, as functions of the temperature $T$ at constant density. According to Fig. 7(a), the role of the interaction component in the temperature elevation is to increase $\beta \Delta \mu$ and reduce the affinity of methane. On the other hand, it is seen in Fig. 7 (b) that $\beta\left(\Delta \mu-\left\langle u_{\mathrm{uv}}\right\rangle\right)$ is a weakly decreasing function of the temperature $T$. Thus, Figs. 5 and 7 imply that while the contribution of $\left\langle u_{\mathrm{uv}}\right\rangle$ to the $\Delta \mu$ value is smaller at a higher temperature, the temperature dependence of $\beta \Delta \mu$ is dominated by that of $\beta\left\langle u_{\mathrm{uv}}\right\rangle$. In other words, the reduced affinity of methane at higher temperatures results from the weakened attraction between the solute and solvent.

The density dependence of the excess chemical potential $\Delta \mu$ of methane in the supercritical conditions can also be analyzed by decomposing $\Delta \mu$ into the interaction and cavity components. In Fig. 8, we show $\left\langle u_{\text {uv }}\right\rangle$ and $\left(\Delta \mu-\left\langle u_{\text {uv }}\right\rangle\right)$ as functions of the water density $\rho$ at a temperature of $400{ }^{\circ} \mathrm{C}$, along with the reference values at the ambient state (the state $A$ in Table I). The density dependence of the interaction component $\left\langle u_{\mathrm{uv}}\right\rangle$ is not monotonic at a fixed temperature. In the density region up to $\sim 0.8 \mathrm{~g} / \mathrm{cm}^{3},\left\langle u_{\text {uv }}\right\rangle$ becomes more attractive (more negative) with the density, and simply increases in magnitude with the number of solvent molecules around the solute. When the density is higher than $\sim 0.8 \mathrm{~g} / \mathrm{cm}^{3}$, water penetrates more into the repulsive core of methane and $\left\langle u_{\mathrm{uv}}\right\rangle$ becomes less attractive with the density. On the other hand, the cavity component $\left(\Delta \mu-\left\langle u_{\mathrm{uv}}\right\rangle\right)$ is more repulsive (more positive) at a higher density over the whole region of density shown in Fig. 8. Therefore, when the 


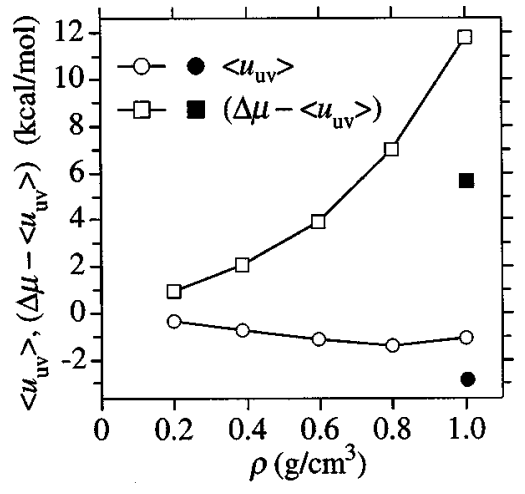

FIG. 8. The interaction component $\left\langle u_{\mathrm{uv}}\right\rangle$ and the cavity component $(\Delta \mu$ $\left.-\left\langle u_{\mathrm{uv}}\right\rangle\right)$ of the hydration thermodynamics of methane as functions of the density $\rho$ at the ambient state $A$ and at the supercritical states $E, F, G, H$, and $I$. The filled symbols represent the state $A$ and the open symbols connected by solid lines represent the states $E, F, G, H$, and $I$. The error bar is smaller than the size of the corresponding symbol.

density is lower than $\sim 0.8 \mathrm{~g} / \mathrm{cm}^{3}$, the interaction and cavity components compete against each other and the density dependence of $\Delta \mu$ is determined by that of the cavity component.

In Fig. 6(a), it is seen that the free energy penalty $\Delta \mu$ of methane insertion is not smaller at the medium-density states $F$ and $G$ in the supercritical region than at the ambient state $A$. Figure 8 shows, however, that the cavity component $\left(\Delta \mu-\left\langle u_{\mathrm{uv}}\right\rangle\right)$ obtained by excluding the interaction component is smaller at the state $G$ than at the state $A$. In other words, when the system is moved from the state $A$ to $G$, the reduction in the cavity component is compensated by the unfavorable change in the solute-solvent interaction $\left\langle u_{\text {uv }}\right\rangle$ and does not give rise to a favorable change in the free energy penalty $\Delta \mu$. When the comparison is made between the states $A$ and $F$, on the other hand, $\left(\Delta \mu-\left\langle u_{\mathrm{uv}}\right\rangle\right)$ is still larger at the state $F$. In this case, both the interaction and cavity components are more repulsive at the supercritical state $F$ than at the ambient state $A$, and the enhanced affinity at the state $F$ is accounted for only by incorporating the factor $\beta$.

In Sec. V, we examine $\beta \Delta \mu$ of the hard sphere solute as a function of its exclusion radius $\lambda$. In this connection, it is of interest to estimate the effective hard core radius $\lambda_{\text {eff }}$ of the methane-water interaction. At each thermodynamic state of interest in Table I, we determine $\lambda_{\text {eff }}$ from the simulation

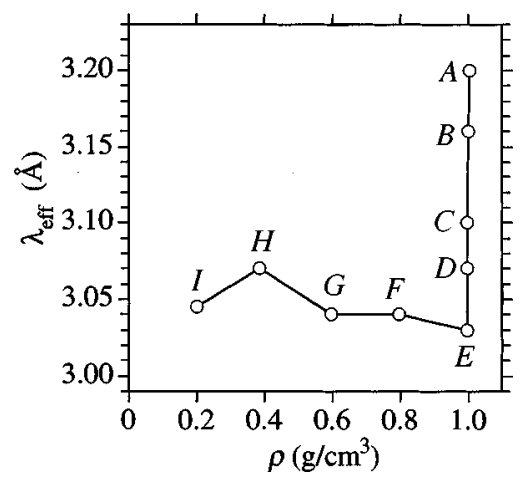

FIG. 9. The effective hard core radius $\lambda_{\text {eff }}$ of the methane-water interaction as a function of the density $\rho$ at the thermodynamic states listed in Table I. results in Sec. V so that $\beta \Delta \mu$ of the hard sphere solute of exclusion radius $\lambda_{\text {eff }}$ is equal to $\beta\left(\Delta \mu-\left\langle u_{\mathrm{uv}}\right\rangle\right)$ of methane. In Fig. 9, we show the $\lambda_{\text {eff }}$ thus determined as a function of the density $\rho$ at the thermodynamic states listed in Table I. It is seen that at each state, $\lambda_{\text {eff }}$ corresponds to the sum of the van der Waals radii of methane and water. When the (water) density is fixed at $1.0 \mathrm{~g} / \mathrm{cm}^{3}, \lambda_{\text {eff }}$ decreases with the temperature $T$. In addition, it is found in the accompanying paper that the first peak of the methane-water radial distribution function is shifted to a smaller distance by the temperature elevation in the constant volume condition. ${ }^{47}$ Thus, when the temperature is raised at constant density, water penetrates "deeper" into the core of methane and $\lambda_{\text {eff }}$ becomes smaller. Actually, we show in Sec. V that $\beta \Delta \mu$ of a hard sphere solute increases with the temperature when its exclusion radius $\lambda$ is fixed. This implies that the weak decrease in $\beta\left(\Delta \mu-\left\langle u_{\mathrm{uv}}\right\rangle\right)$ of methane against the temperature elevation seen in Fig. 7(b) is due to the reduction in $\lambda_{\text {eff }}$, which is in turn connected to the softness of the solute-solvent interaction. The density dependence of $\lambda_{\text {eff }}$ is relatively small. In other words, the effective hard core radius for the nonpolar solute is essentially determined by the temperature.

\section{THERMODYNAMICS OF CAVITY FORMATION}

In fluid, it is always possible to find cavities of various sizes. The probability of finding a cavity is related through Eq. (3) to the excess chemical potential of the corresponding hard sphere solute. ${ }^{39,41,43,46}$ Since the hard sphere is a prototypical nonpolar solute, it is useful to examine the cavity distribution in pure solvent water at high-temperature conditions. In this section, we focus on the thermodynamics of cavity formation over a wide range of density and temperature.

The excess chemical potential $\Delta \mu$ of the hard sphere solute of exclusion radius $\lambda$ is determined from Eq. (3) by the probability $p(\lambda)$ of successful insertion of the hard sphere. As noted in Sec. II, $p(\lambda)$ denotes the probability that the spherical solute is separated from any water molecule by a distance of at least $\lambda$ when it is inserted at an arbitrary position in the fluid. In other words, $p(\lambda)$ is the probability that an arbitrarily chosen position in the fluid is the center of a cavity whose radius is at least $\lambda$. In order to analyze the microscopic inhomogeneity of the pure solvent, Pohorille and Pratt introduced $p_{m}(\lambda)$ defined as ${ }^{41,43}$

$$
p(\lambda)=\int_{\lambda}^{\infty} d \lambda^{\prime} p_{m}\left(\lambda^{\prime}\right) .
$$

$p_{m}(\lambda)$ is the probability distribution function that the radius of a cavity at an arbitrarily chosen position in the fluid is equal to $\lambda$. Using $p_{m}(\lambda)$, it is possible to characterize the range of the size of a cavity which appears spontaneously and transiently in the fluid.

In Fig. 10(a), we show $\beta \Delta \mu$ of the hard sphere solute as a function of its exclusion radius $\lambda$ at the thermodynamic states involving water densities of $1.0 \mathrm{~g} / \mathrm{cm}^{3}$ (the states $A, B$, $C, D$, and $E$ in Table I). According to Fig. 10 (a), $\beta \Delta \mu$ increases with the temperature at each $\lambda$, though the temperature dependence is weak. ${ }^{117}$ In other words, a cavity of mo- 

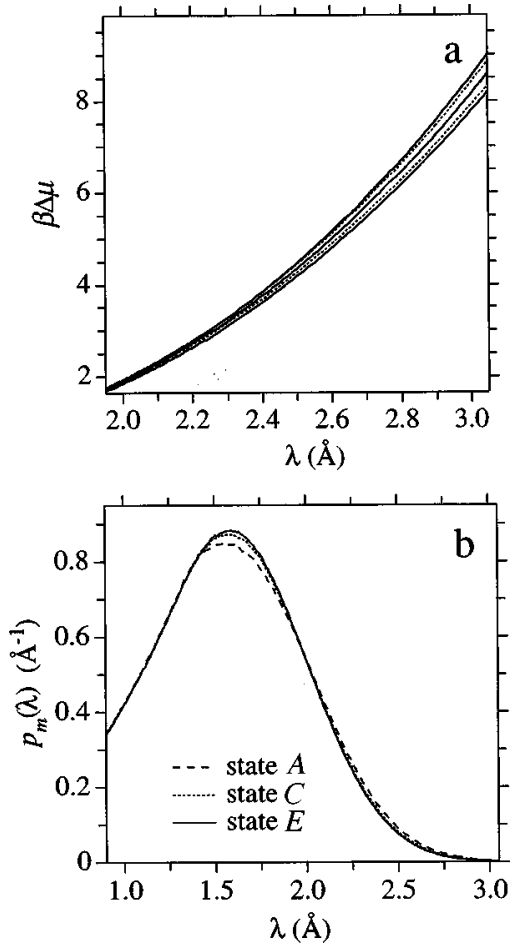

FIG. 10. (a) $\beta \Delta \mu$ of cavity formation as a function of the cavity radius $\lambda$ at the states $A, B, C, D$, and $E$, which involve water densities of $1.0 \mathrm{~g} / \mathrm{cm}^{3}$. The solid lines represent the states $A, C$, and $E$, and the dotted lines represent the states $B$ and $D$. At each $\lambda, \beta \Delta \mu$ corresponds to the states $E, D, C$, $B$, and $A$ from top to bottom. (b) The probability distribution function $p_{m}(\lambda)$ of the cavity size as a function of the cavity radius $\lambda$ at the states $A, C$, and $E$. The dashed line represents the state $A$, the dotted line represents the state $C$, and the solid line represents the state $E$.

lecular size $(\lambda \gtrsim 2.0 \AA)$ becomes less likely to be found in water when the temperature is elevated in the constant volume condition. In previous computer simulations at constant density, Pohorille and Pratt and Ikeguchi et al. found that $\beta \Delta \mu$ increases when the temperature is raised from $\sim 25$ to $\sim 100^{\circ} \mathrm{C} .{ }^{41,43,46}$ Figure 10 (a) actually shows that the increasing behavior of $\beta \Delta \mu$ is observed even in the supercritical region.

To see the relationship between the temperature dependence of $\beta \Delta \mu$ and the local inhomogeneities of the pure solvent system, in Fig. 10(b) we show $p_{m}(\lambda)$ defined by Eq. (9) at the states $A, C$, and $E$. It is evident in the small $\lambda$ region $(\lambda \lesssim 1.5 \AA)$ that $p_{m}(\lambda)$ is essentially indistinguishable among the states involving the same density but different temperatures. This simply reflects the fact that the formation of a small cavity is determined only by the density of the pure solvent system..$^{23-25,41,43}$ When the cavity size is larger $(\lambda \gtrsim 1.5 \AA)$, on the other hand, $p_{m}(\lambda)$ varies with the temperature according to the change in the solvent structure shown in Fig. 1. In this region of $\lambda$, the cavity is more sharply distributed at a higher temperature and $p_{m}(\lambda)$ decreases with the temperature for cavities of molecular size $(\lambda \geqslant 2.0 \AA)$. By comparing water and organic solvents in ambient conditions, Pohorille and Pratt argued that since $p_{m}(\lambda)$ is sharper in water, $\beta \Delta \mu$ is larger in water for a cavity of molecular size. ${ }^{41,43,46}$ In Fig. 10(b), the roles of water and the organic solvents in Pohorille and Pratt's argu-
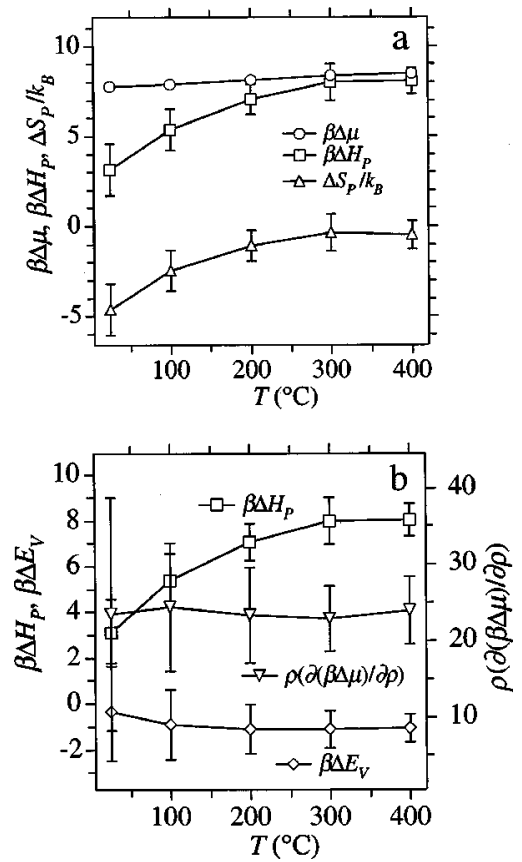

FIG. 11. (a) $\beta \Delta \mu$ and its enthalpic and entropic components $\beta \Delta H_{P}$ and $\Delta S_{P} / k_{B}$ of the hard sphere solute of exclusion radius $3.0 \AA$ as functions of the temperature $T$ at the states $A, B, C, D$, and $E$, which involve water densities of $1.0 \mathrm{~g} / \mathrm{cm}^{3}$. (b) $\beta \Delta H_{P}, \beta \Delta E_{V}$, and $\rho(\partial(\beta \Delta \mu) / \partial \rho)$ of the hard sphere solute of exclusion radius $3.0 \AA$ as functions of the temperature $T$ at the states $A, B, C, D$, and $E$. When not shown, the error bar is smaller than the size of the corresponding symbol.

ments are reversed. Although water is more like a simple liquid at higher temperatures as seen from the radial distribution function presented in Fig. 1(a), the temperature elevation sharpens the distribution of the cavity size and leads to larger $\beta \Delta \mu$ for cavities of molecular size.

The change in $\beta \Delta \mu$ is, however, less than $10 \%$ for each $\lambda$ shown in Fig. 10(a) even when the temperature is raised from $25^{\circ} \mathrm{C}$ (the state $A$ ) to $400{ }^{\circ} \mathrm{C}$ (the state $E$ ). This change in $\beta \Delta \mu$ is actually smaller by an order of magnitude than the corresponding changes in the enthalpic and entropic components $\beta \Delta H_{P}$ and $\Delta S_{P} / k_{B}$. In Fig. 11(a), we show $\beta \Delta \mu$, $\beta \Delta H_{P}$, and $\Delta S_{P} / k_{B}$ as functions of the temperature $T$ at a fixed water density of $1.0 \mathrm{~g} / \mathrm{cm}^{3}$ for a selected cavity radius of $\lambda=3.0 \AA$. The results are shown only at $\lambda=3.0 \AA$ for brevity since the thermodynamic characteristics in Fig. 11(a) are valid over the range of $\lambda$ in Fig. 10(a). It is indeed seen over a wide range of temperature that the large changes in $\beta \Delta H_{P}$ and $\Delta S_{P} / k_{B}$ compensate each other and lead to the small change in $\beta \Delta \mu$.

When the response of $\beta \Delta \mu$ to temperature variation is weak at constant density, the temperature dependence of $\beta \Delta H_{P}$ can be related to that of the thermal expansion coefficient $\alpha_{P}$ of the pure solvent. To see this, we note the thermodynamic identity that

$$
\beta \Delta H_{P}=\beta\left(\Delta E_{V}+\frac{T \alpha_{P}}{\kappa_{T}} \Delta V\right)=\beta \Delta E_{V}+T \alpha_{P} \rho \frac{\partial(\beta \Delta \mu)}{\partial \rho},
$$

where $\Delta E_{V}$ is the excess partial molar energy at constant volume, $\Delta V$ is the excess partial molar volume, and $\kappa_{T}$ is the 
isothermal compressibility of pure water. $\Delta E_{V}$ can be obtained from the dependence of $\beta \Delta \mu$ on the temperature $T$ at constant density shown in Figs. 10(a) and 11(a). In this work, we estimate $\beta \Delta E_{V}$ by fitting $\beta \Delta \mu$ to a quadratic function of $1 / T$ and using Eq. (7). In Fig. 11(b), we show $\beta \Delta H_{P}$, $\beta \Delta E_{V}$, and $\rho(\partial(\beta \Delta \mu) / \partial \rho)$ as functions of the temperature $T$ at a fixed water density of $1.0 \mathrm{~g} / \mathrm{cm}^{3}$ for a cavity with the radius of $\lambda=3.0 \AA$. The trend in Fig. 11(b) is valid over the range of $\lambda$ in Fig. 10(a), and we show only the results at $\lambda$ $=3.0 \AA$ for brevity. It is then seen that $\beta \Delta H_{P}$ is dominated by the second term of Eq. (10). In addition, Fig. 11(b) suggests that $\rho(\partial(\beta \Delta \mu) / \partial \rho)$ is a weak function of $T$, which is consistent with the observation that the temperature dependence of $\beta \Delta \mu$ is weak in the constant volume condition. Thus, it is concluded on the basis of Eq. (10) that the temperature dependence of $\beta \Delta H_{P}$ is parallel to that of $T \alpha_{P}$ presented in Fig. 3(b). In the temperature range from $\sim 25$ to $\sim 100^{\circ} \mathrm{C}$, Ikeguchi et al. also emphasized the parallelism in the temperature dependence between $\beta \Delta H_{P}$ and $T \alpha_{P} \cdot{ }^{38,44,46}$ Our results then show that when the volume of the system is fixed, the parallelism caused by the weak temperature dependence of $\beta \Delta \mu$ is valid over a wide temperature range including the supercritical states. In this case, a larger $T \alpha_{P}$ leads to a larger $\beta \Delta H_{P}$.

According to Fig. 10(a), $\beta \Delta \mu$ exhibits a weak but appreciable dependence on the temperature $T$ in the constant volume condition. In order to identify the factor determining the $T$ dependence of $\beta \Delta \mu$, we perform the component analysis for the thermodynamics of cavity formation within the framework of the scaled-particle theory. The thermodynamics of cavity formation can be represented in analytically convenient form by the scaled-particle theory. ${ }^{23-25}$ In this theory, the detailed interaction and structure of the solution are not taken into account explicitly and affect the free energy of cavity formation only through the solvent density $\rho$, pressure $P$, and temperature $T$ of the system. The solvent molecule is treated as a hard sphere with a properly chosen diameter $\sigma_{v}$, and the insertion of a cavity of radius $\lambda$ is equivalent to that of a solute molecule of diameter $2 \lambda$ $-\sigma_{v}$. Since we are concerned with cavities of molecular size, we restrict our attention to the solutes which involve positive diameters and satisfy $\lambda \geqslant \sigma_{v} / 2$. The free energy of cavity formation $\Delta \mu^{\mathrm{SPT}}$ is then expressed in the scaledparticle theory as ${ }^{23,25}$

$$
\begin{aligned}
\beta \Delta \mu^{\mathrm{SPT}}= & -\ln (1-y)+\left(\frac{3 y}{1-y}\right) R \\
& +\left[\frac{3 y}{1-y}+\frac{9}{2}\left(\frac{3 y}{1-y}\right)^{2}\right] R^{2}+\frac{\beta y P}{\rho} R^{3},
\end{aligned}
$$

where $y$ is the packing fraction of the solvent defined as

$$
y=\frac{\pi}{6} \rho \sigma_{v}^{3}
$$

and $R$ is the ratio of the solute diameter to the solvent diameter given by

$$
R=\frac{2 \lambda-\sigma_{v}}{\sigma_{v}}
$$

TABLE II. The effective diameter of the water molecule and the accuracy of the scaled-particle theory for the free energy of cavity formation.

\begin{tabular}{ccccc}
\hline \hline State & $\begin{array}{c}\lambda_{\min } \\
(\AA)\end{array}$ & $\begin{array}{c}\lambda_{\max } \\
(\AA)\end{array}$ & $\begin{array}{c}\sigma_{v} \\
(\AA)\end{array}$ & $\delta_{\min }$ \\
\hline$A$ & 2.0 & 3.0 & 2.81 & $1.1 \times 10^{-1}$ \\
$B$ & 2.0 & 3.0 & 2.80 & $7.6 \times 10^{-2}$ \\
$C$ & 2.0 & 3.0 & 2.79 & $5.2 \times 10^{-2}$ \\
$D$ & 2.0 & 3.0 & 2.77 & $4.3 \times 10^{-2}$ \\
$E$ & 2.0 & 3.0 & 2.76 & $3.8 \times 10^{-2}$ \\
$F$ & 2.0 & 4.0 & 2.74 & $1.2 \times 10^{-2}$ \\
$G$ & 2.0 & 5.0 & 2.57 & $7.5 \times 10^{-2}$ \\
$H$ & 2.0 & 7.0 & 2.04 & $1.6 \times 10^{-1}$ \\
$I$ & 2.0 & 9.0 & 1.72 & $7.8 \times 10^{-2}$ \\
$\widetilde{G}$ & 2.0 & 5.0 & 2.64 & $5.9 \times 10^{-2}$ \\
$\widetilde{H}$ & 2.0 & 7.0 & 2.30 & $9.7 \times 10^{-2}$ \\
$\widetilde{I}$ & 2.0 & 9.0 & 2.19 & $5.3 \times 10^{-2}$ \\
\hline \hline
\end{tabular}

In our treatments, $\rho, P$, and $T$ are taken from Table I and the exact equation of state for the system under study (SPC/E water) is employed in Eq. (11). When the solvent is water, $\sigma_{v}$ is actually an effective diameter of the solvent molecule. In this case, $\sigma_{v}$ can be considered an adjustable parameter in Eq. (11) and may be treated as a function of the state of the system.

The accuracy of the approximate expression Eq. (11) is assessed from the average deviation $\delta$ defined as

$$
\delta=\frac{1}{\lambda_{\max }-\lambda_{\min }} \int_{\lambda_{\min }}^{\lambda_{\max }} d \lambda\left|\beta \Delta \mu^{\mathrm{SPT}}-\beta \Delta \mu\right|,
$$

where $\Delta \mu$ is the exact free energy of cavity formation obtained from the simulations and $\lambda_{\min }$ and $\lambda_{\max }$ are the smallest and largest radii of the cavities of interest, respectively. In this work, we determine the effective diameter $\sigma_{v}$ of the water molecule by minimizing $\delta$ with respect to $\sigma_{v}$. At each state connected to the isochoric thermodynamic path from the state $A$ to $E$, we take $\lambda_{\min }=2.0 \AA$ and $\lambda_{\max }=3.0 \AA$ and obtain the $\sigma_{v}$ which minimizes $\delta$. Table II shows the $\sigma_{v}$ thus obtained and the minimized value $\delta_{\min }$ of the deviation $\delta$ at the thermodynamic states listed in Table I. It is seen that when the water density is fixed at $1.0 \mathrm{~g} / \mathrm{cm}^{3}$, the effective diameter $\sigma_{v}$ of the water molecule corresponds to the van der Waals diameter of water and is close to the values adopted by Pierotti at ambient conditions ${ }^{23,25}$ and by Crovetto et al. on the liquid branch of the saturation curve up to a temperature of $\sim 250{ }^{\circ} \mathrm{C} .{ }^{49,50}$ The temperature dependence of $\sigma_{v}$ is weak, and $\sigma_{v}$ decreases only by $\sim 2 \%$ when the temperature is elevated from 25 to $400{ }^{\circ} \mathrm{C}$. As an approximate expression for the free energy of cavity formation, $\Delta \mu^{\mathrm{SPT}}$ given by Eq. (11) involves an error of $\sim 0.1 k_{B} T$ at the ambient state (the state $A$ ) and its accuracy improves with the temperature.

At ambient conditions, the effect of the pressure is negligible in the free energy of cavity formation. When the temperature is raised at constant density, the pressure increases by orders of magnitude as shown in Table I, and the pressure effect may be significant in the thermodynamics of cavity formation. In the scaled-particle theory, the pressure effect on the free energy of cavity formation $\Delta \mu^{\mathrm{SPT}}$ is represented by the last term of Eq. (11). It is cubic with respect to the 


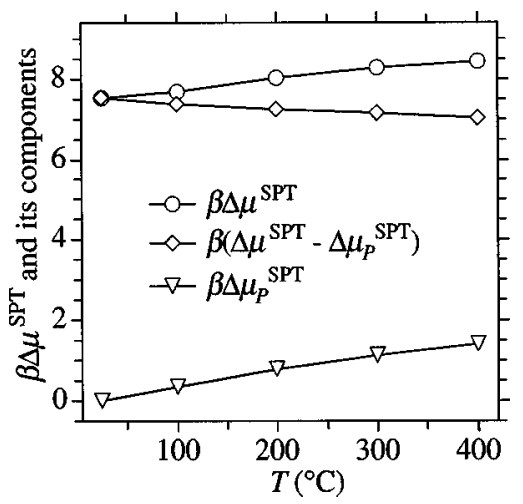

FIG. 12. $\beta \Delta \mu^{\mathrm{SPT}}$ and its components $\beta \Delta \mu_{P}^{\mathrm{SPT}}$ and $\beta\left(\Delta \mu^{\mathrm{SPT}}-\Delta \mu_{P}^{\mathrm{SPT}}\right)$ evaluated from the scaled-particle theory for the hard sphere solute of exclusion radius $3.0 \AA$ as functions of the temperature $T$ at the states $A, B, C$, $D$, and $E$, which involve water densities of $1.0 \mathrm{~g} / \mathrm{cm}^{3}$.

ratio $R$ of the solute diameter to the solvent diameter and proportional to the pressure $P$. The density $\rho$ and the packing fraction $y$ do not have to be referred explicitly in the pressure effect since they are related to each other through Eq. (12). Let $\Delta \mu_{P}^{\mathrm{SPT}}$ denote $y P R^{3} / \rho$, which represents the pressure term in Eq. (11). $\beta\left(\Delta \mu^{\mathrm{SPT}}-\Delta \mu_{P}^{\mathrm{SPT}}\right)$ is then independent of the pressure $P$ explicitly and is determined only by the packing fraction $y$ and the diameter ratio $R$. In Fig. 12, we show $\beta \Delta \mu^{\mathrm{SPT}}$ and its components $\beta \Delta \mu_{P}^{\mathrm{SPT}}$ and $\beta\left(\Delta \mu^{\mathrm{SPT}}\right.$ $\left.-\Delta \mu_{P}^{\mathrm{SPT}}\right)$ as functions of the temperature $T$ at a fixed water density of $1.0 \mathrm{~g} / \mathrm{cm}^{3}$ for a cavity with the radius of $\lambda=3.0$ $\AA$. The general trend observed in Fig. 12 holds over the range of $\lambda$ in Fig. 10(a), and the results are shown only at $\lambda=3.0 \AA$ for brevity. It is then seen in Fig. 12 that $\beta\left(\Delta \mu^{\mathrm{SPT}}-\Delta \mu_{P}^{\mathrm{SPT}}\right)$ decreases with $T$. This is caused by the reduction in the effective diameter $\sigma_{v}$ of the water molecule. At a higher temperature, $\sigma_{v}$ is smaller and the solvent is considered less packed. On the other hand, the pressure term $\beta \Delta \mu_{P}^{\mathrm{SPT}}$ is an increasing function of $T$. This increasing behavior is dominated entirely by the response of $P / T$ to the temperature variation, while the effect of the $\sigma_{v}$ reduction is negligible in the pressure term. According to Fig. (12), furthermore, $\beta \Delta \mu_{P}^{\mathrm{SPT}}$ is a stronger function of $T$ than $\beta\left(\Delta \mu^{\mathrm{SPT}}-\Delta \mu_{P}^{\mathrm{SPT}}\right)$ and the temperature dependence of $\beta \Delta \mu^{\mathrm{SPT}}$ is governed by the pressure term. Thus, when the temperature is raised at constant density, the accompanying pressure elevation reduces the probability of finding a cavity of molecular size in water.

We now turn to the density dependence of the cavity distribution in the supercritical states. The free energy $\Delta \mu$ of formation of a cavity of radius $\lambda$ is related through Eq. (3) to the probability $p(\lambda)$ of finding the cavity. In Fig. 13(a), we show $\beta \Delta \mu$ as a function of the cavity radius $\lambda$ at the thermodynamic states involving temperatures of $400{ }^{\circ} \mathrm{C}$ (the states $E, F, G, H$, and $I$ in Table I). The overall behavior of $\beta \Delta \mu$ [or $p(\lambda)]$ is similar to that characterized by Mountain in the region on which both our and his works focus. ${ }^{118}$ According to Fig. 13(a), $\beta \Delta \mu$ increases monotonically with the density at each $\lambda$, and a hard sphere solute involves a higher affinity for lower-density water. In order to characterize the microscopic inhomogeneity of the pure solvent in connection to the thermodynamics of cavity formation, it is
N. Matubayasi and M. Nakahara
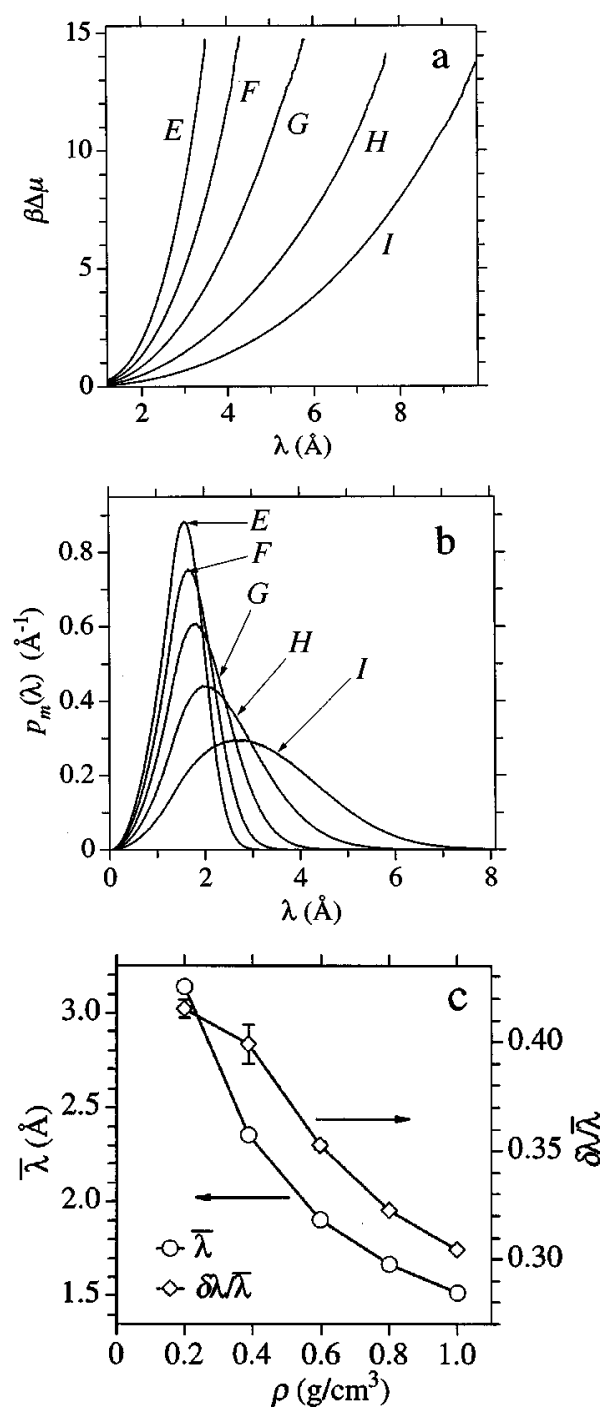

FIG. 13. (a) $\beta \Delta \mu$ of cavity formation as a function of the cavity radius $\lambda$ at the supercritical states $E, F, G, H$, and $I$. At each $\lambda, \beta \Delta \mu$ corresponds to the states $E, F, G, H$, and $I$ from top to bottom. (b) The probability distribution function $p_{m}(\lambda)$ of the cavity size as a function of the cavity radius $\lambda$ at the supercritical states $E, F, G, H$, and $I$. (c) The average size of the cavity $\bar{\lambda}$ and the relative deviation $\delta \lambda / \bar{\lambda}$ as functions of the density $\rho$ at the supercritical states $E, F, G, H$, and $I$. In (c), the error bar is smaller than the size of the corresponding symbol when it is not shown.

insightful to examine the probability distribution function $p_{m}(\lambda)$ of the cavity radius introduced by Eq. (9). In Fig. 13(b), we show $p_{m}(\lambda)$ as a function of $\lambda$ at the states $E, F$, $G, H$, and $I$. It is evident that $p_{m}(\lambda)$ is concentrated in the larger $\lambda$ region when the density is lower. This simply shows that the cavity is likely to be larger at a lower density. Furthermore, the width of the distribution function $p_{m}(\lambda)$ reduces with the density and the cavity size is more uniform at a higher density. Using the distribution function $p_{m}(\lambda)$, the average size of the cavity $\bar{\lambda}$ and the standard deviation $\delta \lambda$ can be evaluated by

$$
\begin{aligned}
& \bar{\lambda}=\int d \lambda \lambda p_{m}(\lambda), \\
& \delta \lambda=\sqrt{\int d \lambda \lambda^{2} p_{m}(\lambda)-\bar{\lambda}^{2}} .
\end{aligned}
$$


Figure 13(c) shows $\bar{\lambda}$ and $\delta \lambda / \bar{\lambda}$ as functions of the density $\rho$ at a fixed supercritical temperature of $400{ }^{\circ} \mathrm{C}$. It is indeed seen that $\bar{\lambda}$ decreases with $\rho$. The decreasing rate is larger in the lower-density region. This is actually consistent with the fact that $\bar{\lambda}$ is divergent in the limit of zero density. The density reduction also enhances the inhomogeneity of the cavity size represented by $\delta \lambda / \bar{\lambda}$. In Sec. III, it has been observed that the spatial distribution of the water molecules is more inhomogeneous at a lower density. In other words, the contrast between the dense and sparse regions of the water molecules is stronger when the density is lower. Figure 13(c) implies furthermore that the dense and sparse regions are more scattered in size in a lower-density fluid.

The effective diameter $\sigma_{v}$ of the water molecule can be estimated by fitting $\beta \Delta \mu$ in Fig. 13(a) to Eq. (11) on the basis of the scaled-particle theory. The scheme to determine $\sigma_{v}$ at the thermodynamic states connected to the isothermal path from the state $E$ to $I$ is the same as that used above at the states connected to the isochoric path from the state $A$ to $E$. At each thermodynamic state, we employ $\lambda_{\min }$ and $\lambda_{\max }$ shown in Table II and minimize the average deviation $\delta$ defined by Eq. (14) with respect to $\sigma_{v}$. In Table II, we show the $\sigma_{v}$ thus determined and the minimized value $\delta_{\min }$ of the deviation $\delta$ at the thermodynamic states listed in Table I. It is seen at a fixed temperature of $400{ }^{\circ} \mathrm{C}$ that $\sigma_{v}$ decreases when the density is reduced. Especially, when the density is lower than $\sim 0.6 \mathrm{~g} / \mathrm{cm}^{3}$, the van der Waals diameter of the water molecule is not effective to estimate $\sigma_{v}$. According to Eq. (11), even when $\sigma_{v}$ is independent of the density, the free energy $\Delta \mu$ of cavity formation will be smaller at a lower density. The response of $\Delta \mu$ to the density is then further strengthened by the density dependence of $\sigma_{v}$ shown in Table II. The magnitude of $\sigma_{v}$ is related to the extent of spatial inhomogeneity of pure water described in Sec. III. When the pure solvent system is spatially more inhomogeneous, a larger cavity is likely to be found and the solvent molecule is effectively smaller. In Appendix A, this point is further pursued in connection to the state dependence of the dipole moment of a water molecule. When the accuracy of the scaled-particle theory is concerned, Table II shows that $\Delta \mu^{\mathrm{SPT}}$ given by Eq. (11) is an approximate expression for the free energy of cavity formation at the supercritical states with errors on the order of $0.1 k_{B} T$. Therefore, the scaledparticle theory accurately represents the thermodynamics of cavity formation in supercritical water when the effective diameter of the water molecule is properly chosen.

Since a simple and analytic expression for the excess partial molar enthalpy is obtained from the temperature differentiation of Eq. (11), the scaled-particle theory also provides a convenient framework for describing the excess partial molar enthalpy. In Appendix C, we analyze the density and temperature dependence of the excess partial molar enthalpy within the framework of the scaled-particle theory.

\section{CONCLUSIONS}

The excess chemical potential and its enthalpic and entropic components have been analyzed for the methane and hard sphere solutes in water over a wide range of thermody- namic conditions. It has been found that the free energy penalty of inserting the nonpolar solute is larger at the superand subcritical conditions with the medium to high density than at the ambient condition. In this case, the enhanced affinity of the nonpolar solute for super- and subcritical water results from the elevated temperature, which is a nonspecific driving force for mixing any two materials. When the enthalpic and entropic components are compared between the ambient and high-temperature conditions, it has been shown that the nonpolar solute in high-temperature water is destabilized by the enthalpic component and is stabilized by the entropic component. In connection to the thermodynamics of cavity formation, the size distribution of spherical cavities has also been examined in pure solvent water. When the density is fixed at a liquidlike value, it has been found that the probability of finding a cavity of molecular size in water is a weakly decreasing function of the temperature. This has then been shown, in the framework of the scaledparticle theory, to result from the elevated pressure involved at high temperatures. When the density is varied at a fixed supercritical temperature, it has been observed that the size distribution is less uniform at a lower density. The effective diameter of the water molecule has been estimated within the framework of the scaled-particle theory, and its reduction at a lower density has been related to the extent of spatial inhomogeneity in pure solvent water.

\section{ACKNOWLEDGMENTS}

This work is supported by the Research Grant-in-Aid from the Ministry of Education, Science, and Culture (No. 10304047) and by CREST (Core Research for Evolutional Science and Technology) of Japan Science and Technology Corporation (JST). N. M. is also grateful to the Research Grant-in-Aid from the Ministry of Education, Science, and Culture (No. 11740322) and to the Supercomputer Laboratory of Institute for Chemical Research, Kyoto University for generous allocation of computation time.

\section{APPENDIX A}

In the SPC/E model employed in this work, the dipole moment of a water molecule is a fixed parameter and does not depend on the density and temperature of the system. However, the dipole moment in the SPC/E model is tuned to reproduce ambient liquid properties of water and is inappropriate to simulate the dilute gas of water. Thus, the dipole moment needs to be treated as a function of the thermodynamic state in order to perform realistic computer simulations of water over a wide range of thermodynamic conditions. In practical implementations of computer simulations, there are two methods to incorporate the state-dependent nature of the dipole moment into the potential model of water. One is to employ a polarizable model and determine the charge distribution within a water molecule at each step of the simulation. ${ }^{81,84-94}$ In this method, although no ad hoc parameters are required in principle, a set of potential parameters which covers a wide range of density and temperature seems yet difficult to find. The other is to construct an effective potential model by fitting some known experimental 
TABLE III. Thermodynamic states of interest in the SPC-like model with a reduced dipole moment.

\begin{tabular}{cccccccc}
\hline \hline & & & \multicolumn{2}{c}{ Canonical ensemble } & & \multicolumn{2}{c}{ Isothermal-isobaric ensemble } \\
State & $\begin{array}{c}\text { Dipole moment } \\
(\text { Debye })\end{array}$ & $\begin{array}{c}\text { Temperature } \\
\left({ }^{\circ} \mathrm{C}\right)\end{array}$ & $\begin{array}{c}\text { Density } \\
\left(\mathrm{g} / \mathrm{cm}^{3}\right)\end{array}$ & $\begin{array}{c}\text { Average pressure } \\
(\mathrm{bar})\end{array}$ & & $\begin{array}{c}\text { Pressure } \\
(\mathrm{bar})\end{array}$ & $\begin{array}{c}\text { Average density } \\
\left(\mathrm{g} / \mathrm{cm}^{3}\right)\end{array}$ \\
\hline$\tilde{G}$ & 2.25 & 400 & 0.60 & $1107 \pm 2$ & & 1107 & 0.60 \\
$\tilde{H}$ & 2.25 & 400 & 0.40 & $520 \pm 2$ & & 520 & 0.40 \\
$\tilde{I}$ & 2.15 & 400 & 0.20 & $381 \pm 1$ & & 381 & 0.20 \\
\hline \hline
\end{tabular}

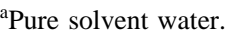

${ }^{\mathrm{b}}$ The values are evaluated in pure solvent water.

${ }^{\mathrm{c}}$ The values are evaluated in pure solvent water. The errors are less than $0.01 \mathrm{~g} / \mathrm{cm}^{3}$.

data. ${ }^{15}$ In this method, although the potential parameters are valid only in a limited region of density and temperature, the simulation results obtained in that region will be reliable.

In Ref. 15, we estimated the dipole moment of a water molecule within the SPC-like framework at densities of 0.6, 0.4 , and $0.2 \mathrm{~g} / \mathrm{cm}^{3}$ and a temperature of $400{ }^{\circ} \mathrm{C}$. In our SPC-like model, the Lennard-Jones parameters and the positions of the charged sites are identical to those of the original models, ${ }^{82,100}$ and the strength of the partial charges, or equivalently, the dipole moment is adjusted to fit the number of hydrogen bonds obtained from the proton chemical shift. ${ }^{13-15}$ The dipole moment given in Ref. 15 is $2.25,2.25$, and $2.15 \mathrm{D}$ at $0.6,0.4$, and $0.2 \mathrm{~g} / \mathrm{cm}^{3}$, respectively, whereas the dipole moment in the SPC/E model is fixed at $2.35 \mathrm{D}$. Thus, the dipole moment is smaller at the supercritical states than at the ambient state. In this Appendix, we analyze the effect of the reduced dipole moment in the SPC-like model on the hydration thermodynamics of nonpolar solutes.

The states to be examined by the SPC-like model are specified by the temperature of $400{ }^{\circ} \mathrm{C}$ and the pressures corresponding to the water densities of $0.6,0.4$, and $0.2 \mathrm{~g} / \mathrm{cm}^{3}$, which are denoted by the states $\widetilde{G}, \widetilde{H}$, and $\widetilde{I}$, respectively. The dipole moment of a water molecule at each state is shown in Table III. The hydration thermodynamics of the methane and hard sphere solutes at the states $\widetilde{G}, \widetilde{H}$, and $\widetilde{I}$ was evaluated from the computer simulations in the isothermal-isobaric ensemble. In order to determine the input pressures for the simulations in the isothermal-isobaric ensemble, we carried out Monte Carlo simulations of pure solvent water in the canonical ensemble with the input densities and temperatures shown in Table III. In each Monte Carlo simulation, 648 water molecules were located in a cubic unit cell and the standard Metropolis sampling scheme was employed. ${ }^{76,96}$ The Monte Carlo simulation was performed for one million passes, and the truncation scheme for the intermolecular interactions and the boundary condition were the same as those for SPC/E water described in Sec. II. The average pressures at the states $\widetilde{G}, \widetilde{H}$, and $\widetilde{I}$ are then shown in Table III. These average pressures are used as the input pressures of the simulations performed in the isothermal-isobaric ensemble. At each of the states in Table III, a Monte Carlo simulation of the pure water system was carried out in the isothermal-isobaric ensemble for one million passes by locating 648 water molecules in a cubic unit cell. $^{76,96}$ The particle insertion method was implemented to evaluate the excess chemical potentials of the methane and hard sphere solutes by employing the parameters presented in Sec. II. The scheme to truncate the intermolecular interactions and the boundary condition were identical to those for the SPC/E model described in Sec. II.

The $\mathrm{O}-\mathrm{O}$ and $\mathrm{O}-\mathrm{H}$ radial distribution functions at the states $\widetilde{G}, \widetilde{H}$, and $\widetilde{I}$ are shown in Fig. 7 of Ref. 15. The radial distribution functions for the SPC/E model at the corresponding states $G, H$, and $I$ in Table I are seen in Fig. 2. When the radial distribution functions are compared between the SPC-like model with a reduced dipole moment and the SPC/E model, their amplitudes are smaller for the model with a smaller dipole moment when the density and temperature are the same. This simply reflects the fact that the attraction between water molecules is stronger when a larger dipole moment is adopted. In Fig. 14, we show the isothermal compressibility $\kappa_{T}$ and the thermal expansion coefficient $\alpha_{P}$ at the states $\widetilde{G}, \widetilde{H}$, and $\widetilde{I}$ as functions of the density $\rho$. It is seen from Figs. 4 and 14 that the thermodynamic response functions related to the density fluctuations are larger for a larger dipole moment. Therefore, the spatial inhomogeneities are smaller, both locally and globally, when the dipole moment is reduced in the SPC-like framework.

Let $\Delta \tilde{\mu}$ denote the excess chemical potential of methane calculated using the SPC-like model with a reduced dipole moment, and let $\Delta \mu$ be that calculated using the SPC/E model. The effect of the dipole moment of a water molecule on the excess chemical potential at fixed density and temperature can be seen by comparing $\Delta \tilde{\mu}$ at the states $\tilde{G}, \tilde{H}$,

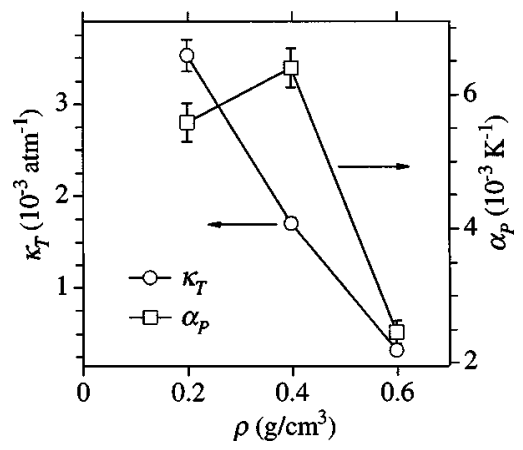

FIG. 14. The isothermal compressibility $\kappa_{T}$ and the thermal expansion coefficient $\alpha_{P}$ as functions of the density $\rho$ at the states $\widetilde{G}, \widetilde{H}$, and $\tilde{I}$, which are simulated using the SPC-like model with a reduced dipole moment. When not shown, the error bar is smaller than the size of the corresponding symbol. 


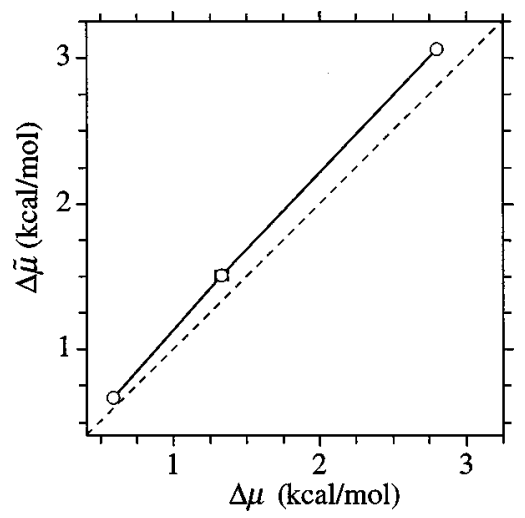

FIG. 15. The excess chemical potential $\Delta \tilde{\mu}$ of methane at the states $\tilde{G}, \tilde{H}$, and $\widetilde{I}$ against the excess chemical potential $\Delta \mu$ of methane at the states $G$, $H$, and $I$. The states $\tilde{G}, \tilde{H}$, and $\tilde{I}$ are simulated using the SPC-like model with a reduced dipole moment, and the states $G, H$, and $I$ are simulated using the SPC/E model. The dashed line represents $\Delta \tilde{\mu}=\Delta \mu$.

and $\widetilde{I}$ with $\Delta \mu$ at the states $G, H$, and $I$, respectively. In Fig. 15 , we make this comparison by plotting $\Delta \tilde{\mu}$ against $\Delta \mu$. Figure 15 shows that when the density and temperature are fixed, the free energy penalty of inserting methane is smaller at a larger dipole moment. This is actually related to the dependence of the extent of spatial inhomogeneity of pure water on the dipole moment. As seen for the radial distribution functions and the thermodynamic response functions, the spatial inhomogeneities are more enhanced when the dipole moment is larger. When the pure solvent system is more inhomogeneous, a cavity of molecular size is more likely to be found and the free energy of its formation will be smaller. To describe the size of spherical cavities in water, Eq. (9) has introduced the distribution function $p_{m}(\lambda)$ as a function of the cavity radius $\lambda$. In Fig. 16, we show $p_{m}(\lambda)$ at the states $\widetilde{G}, \widetilde{H}$, and $\widetilde{I}$ in the SPC-like model with a reduced dipole moment and at the states $G, H$, and $I$ in the SPC/E model. At a fixed density, it is seen that $p_{m}(\lambda)$ is essentially independent of the value of the dipole moment in the small $\lambda$ region. This is simply a reflection of the fact that the probability of finding a sufficiently small cavity is determined only by the

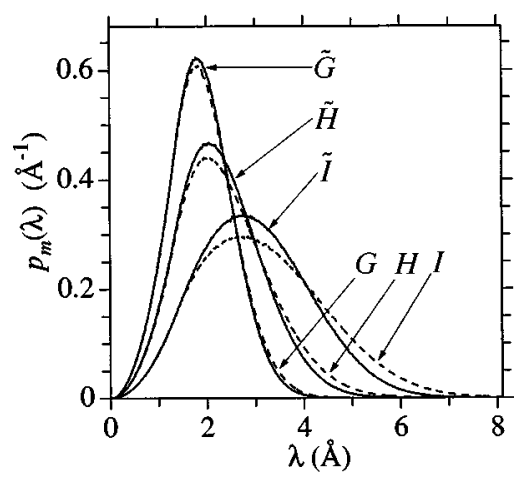

FIG. 16. The probability distribution function $p_{m}(\lambda)$ of the cavity size as a function of the cavity radius $\lambda$ at the states $\widetilde{G}, \widetilde{H}$, and $\widetilde{I}$ and at the states $G$, $H$, and $I$. The solid lines represent the states $\widetilde{G}, \widetilde{H}$, and $\widetilde{I}$, and the dashed lines represent the states $G, H$, and $I$. The states $\widetilde{G}, \widetilde{H}$, and $\widetilde{I}$ are simulated using the SPC-like model with a reduced dipole moment, and the states $G$, $H$, and $I$ are simulated using the SPC/E model. density of the pure solvent system. ${ }^{23-25,41,43}$ When $\lambda \gtrsim 2 \AA$, on the other hand, $p_{m}(\lambda)$ is affected by the value of the dipole moment. When the dipole moment is smaller, the cavity size is more sharply distributed and $p_{m}(\lambda)$ is smaller in the large $\lambda$ region. Therefore, the free energy penalty of inserting a cavity of molecular size is smaller when the dipole moment is larger and the pure solvent system involves more enhanced spatial inhomogeneities.

The probability distribution function $p_{m}(\lambda)$ is related to the free energy $\Delta \mu$ of cavity formation through Eqs. (3) and (9). In Sec. V, we have analyzed $\Delta \mu$ in the framework of the scaled-particle theory and have determined the effective diameter $\sigma_{v}$ of the water molecule. It has then been found that when the spatial inhomogeneity of pure water is enhanced at a lower density, the effective diameter $\sigma_{v}$ is reduced. In order to further pursue the relationship between $\sigma_{v}$ and the solvent structure of water, we also determine the effective diameter $\sigma_{v}$ in the SPC-like model with a reduced dipole moment at the states $\widetilde{G}, \widetilde{H}$, and $\widetilde{I}$. The procedure to determine $\sigma_{v}$ at these states is the same as that described in Sec. $\mathrm{V}$. We employ $\lambda_{\min }$ and $\lambda_{\max }$ shown in Table II and minimize the average deviation $\delta$ defined by Eq. (14) with respect to $\sigma_{v}$. In Table II, we show the $\sigma_{v}$ thus determined and the minimized value $\delta_{\min }$ of the deviation $\delta$ at the thermodynamic states $\widetilde{G}, \widetilde{H}$, and $\widetilde{I}$. It is seen at fixed density and temperature that $\sigma_{v}$ is smaller when the dipole moment of a water molecule is larger. The stronger association among water molecules induces the formation of larger cavities and enhances the probability of finding a cavity of molecular size. Thus, in both cases concerning the variation in the density and in the dipole moment, the effective diameter $\sigma_{v}$ is smaller when the spatial inhomogeneities of water are more enhanced.

\section{APPENDIX B}

In this work, the particle insertion method has been employed to evaluate the excess chemical potentials of the methane and hard sphere solutes. The particle insertion method is a highly efficient method when the solute of interest is successfully inserted at a reasonable rate. ${ }^{76-80}$ Since the rate of successful insertion is too low for a large solute, the validity of the method is limited by the solute size. For purely repulsive solutes in ambient SPC water, Beutler et al. reported that the particle insertion method is accurate only when the thermal radius of the solute-solvent repulsive core does not exceed $3 \AA .{ }^{79}$ In a recent work, on the other hand, Smith examined the particle insertion method in ambient SPC and SPC/E water and found that the method is valid up to the core radius of $4 \AA^{80}{ }^{80}$ In this Appendix, we illustrate the validity of the particle insertion method to calculate the excess chemical potential of methane. To do so, we implement the thermodynamic integration method for methane hydration at the states $A$ and $E$ in Table I and compare the results with those calculated from the particle insertion method. When the particle insertion method is found to be valid at the states $A$ and $E$, it will also be valid at the states connected to the isochoric thermodynamic path from the state $A$ to $E$. When the particle insertion method is shown to be valid at 


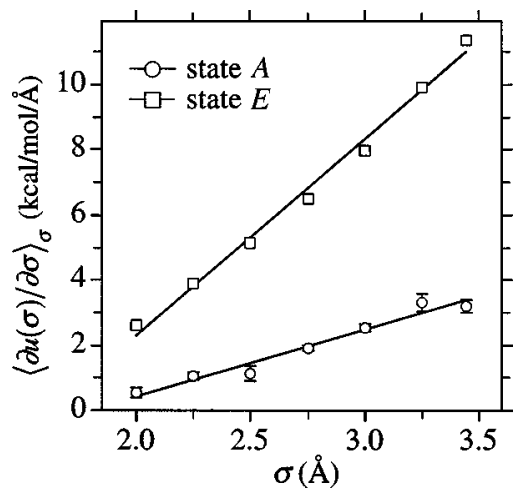

FIG. 17. $\langle\partial u(\sigma) / \partial \sigma\rangle_{\sigma}$ as a function of the Lennard-Jones $\sigma$ at the states $A$ and $E$. The solid lines represent the linear fits (see Ref. 119).

the state $E$, it should be valid at the states connected to the isothermal thermodynamic path from the state $E$ to $I$ since the particle insertion method is a better method at a lower density.

In our thermodynamic integration method to calculate the excess chemical potential of methane, the Lennard-Jones $\epsilon$ is fixed at the value of the full methane-water interaction given in Sec. II and the LJ $\sigma$ is taken as a variable. For a given $\sigma$, we denote the excess chemical potential of the solute and the (instantaneous) sum of the solute-solvent interaction by $\Delta \mu(\sigma)$ and $u(\sigma)$, respectively. When $\sigma$ is varied from $\sigma_{0}$ to the value $\sigma_{\mathrm{Me}-\mathrm{O}}$ at the full methane-water interaction,

$$
\Delta \mu\left(\sigma_{\mathrm{Me}-\mathrm{O}}\right)=\Delta \mu\left(\sigma_{0}\right)+\int_{\sigma_{0}}^{\sigma_{\mathrm{Me}-\mathrm{O}}} d \sigma\left\langle\frac{\partial u(\sigma)}{\partial \sigma}\right\rangle_{\sigma}
$$

holds, where $\langle\cdots\rangle_{\sigma}$ denotes an ensemble average under the presence of the solute-solvent interaction $u(\sigma)$. In Eq. (B1), it is obvious that $\Delta \mu\left(\sigma_{\mathrm{Me}-\mathrm{O}}\right)$ is the excess chemical potential of the (fully coupled) methane solute. We took $\sigma_{0}$ $=2.00 \AA$ and calculated $\Delta \mu\left(\sigma_{0}\right)$ from the particle insertion method in the pure solvent system. In this choice of $\sigma_{0}$, we follow Beutler et al. and consider that the particle insertion method is a valid procedure to calculate $\Delta \mu\left(\sigma_{0}\right) .{ }^{79}$ The procedure for implementing the particle insertion method at $\sigma_{0}$ $=2.00 \AA$ was identical to that described in Sec. II for calculating the excess chemical potential of methane. It was then found that $\Delta \mu\left(\sigma_{0}\right)$ is equal to 0.10 and $1.12 \mathrm{kcal} / \mathrm{mol}$ at the states $A$ and $E$, respectively, with errors of less than $0.01 \mathrm{kcal} / \mathrm{mol}$.

The integrand in Eq. (B1) was calculated at $\sigma=\sigma_{0}$, $2.25,2.50,2.75,3.00,3.25$, and $\sigma_{\mathrm{Me}-\mathrm{O}}$, where the numerals are expressed in the unit of $\AA$. At each $\sigma$, the simulation procedure was the same as that described in Sec. II for aqueous solution of methane. In Fig. 17, we show $\langle\partial u(\sigma) / \partial \sigma\rangle_{\sigma}$ as a function of $\sigma$ at the states $A$ and $E$. When the trapezoidal rule is employed in the integral of Eq. (B1), Fig. 17 gives that $\Delta \mu\left(\sigma_{\mathrm{Me}-\mathrm{O}}\right)=2.88 \pm 0.23$ and $10.67 \pm 0.10 \mathrm{kcal} / \mathrm{mol}$ at the states $A$ and $E$, respectively. ${ }^{119}$ On the other hand, when the particle insertion method is employed, $\Delta \mu\left(\sigma_{\mathrm{Me}-\mathrm{O}}\right)$ is found to be $2.80 \pm 0.07$ and $10.66 \pm 0.02 \mathrm{kcal} / \mathrm{mol}$ at the states $A$ and $E$, respectively. Therefore, at both the states, the excess chemical potentials calculated from the thermody- namic integration method are coincident within the error bars to those calculated from the particle insertion method.

\section{APPENDIX C}

In Sec. V, we have seen that the scaled-particle theory is an accurate representation of the free energy of cavity formation in water over a wide rage of density and temperature when the effective diameter $\sigma_{v}$ of the water molecule is properly chosen in Eq. (11). The scaled-particle theory also provides a convenient framework for describing the excess partial molar enthalpy since a simple and analytic expression for the excess partial molar enthalpy is obtained from the temperature differentiation of Eq. (11). In this Appendix, we analyze the density and temperature dependence of the excess partial molar enthalpy at constant pressure within the framework of the scaled-particle theory.

The expression for the excess partial molar enthalpy at constant pressure $\Delta H_{P}^{\mathrm{SPT}}$ is written in the scaled-particle theory as ${ }^{23,25}$

$$
\begin{aligned}
\beta \Delta H_{P}^{\mathrm{SPT}}= & T \alpha_{P}\left[\frac{y}{1-y}+\frac{3 y}{(1-y)^{2}} R+\frac{3 y(1+2 y)}{(1-y)^{3}} R^{2}\right] \\
& +\frac{\beta y P}{\rho} R^{3}
\end{aligned}
$$

for a cavity with the radius of $\lambda \geqslant \sigma_{v} / 2$, where the packing fraction $y$ of the pure solvent and the ratio $R$ of the solute diameter to the solvent diameter are given by Eqs. (12) and (13), respectively. In our treatments, the solvent density $\rho$, the pressure $P$, the temperature $T$, and the thermal expansion coefficient $\alpha_{P}$ of the pure solvent are taken from Table I and Figs. 3 and 4, and the effective diameter $\sigma_{v}$ of the water molecule listed in Table II is employed. Equation (C1) shows that when $\rho$ and $\sigma_{v}$ are fixed, $\beta \Delta H_{P}^{\text {SPT }}$ increases with $T \alpha_{P}$ and $P / T$. It should be noted that Eq. (C1) does not account for the temperature derivative of $\sigma_{v}$ when it is obtained from the temperature differentiation of Eq. (11) at constant pressure.

According to Eq. (C1), $\beta \Delta H_{P}^{\mathrm{SPT}}$ consists of two terms. The first term is related to the fluctuation in the pure solvent system and is proportional to the thermal expansion coefficient $\alpha_{P}$. The second term $\beta y P R^{3} / \rho$ involves the pressure $P$ of the system and is called the pressure term. Actually, the form of the pressure term is identical to that for the excess chemical potential treated in Sec. V. As done in Sec. V, the pressure term is denoted by $\beta \Delta \mu_{P}^{\mathrm{SPT}}$, and $\beta \Delta \mu_{P}^{\mathrm{SPT}}$ and $\beta\left(\Delta H_{P}^{\mathrm{SPT}}-\Delta \mu_{P}^{\mathrm{SPT}}\right)$ are treated separately as components of $\beta \Delta H_{P}^{\mathrm{SPT}}$.

First, we examine the temperature dependence of the excess partial molar enthalpy at a fixed water density. In Fig. 18(a), we show $\beta \Delta H_{P}^{\mathrm{SPT}}$ and its components as functions of the temperature $T$ at a density of $1.0 \mathrm{~g} / \mathrm{cm}^{3}$ for a cavity with the radius of $\lambda=3.0 \AA$. The results are shown only at $\lambda$ $=3.0 \AA$ for brevity since the trend in Fig. 18(a) is valid over the range of $\lambda$ in Fig. 10(a). According to Fig. 18(a), the pressure term makes a minor contribution to $\beta \Delta H_{P}^{\mathrm{SPT}}$, and $\beta \Delta H_{P}^{\mathrm{SPT}}$ is dominated by the term proportional to $T \alpha_{P}$. In order to isolate the effect of the variation in the solvent di- 

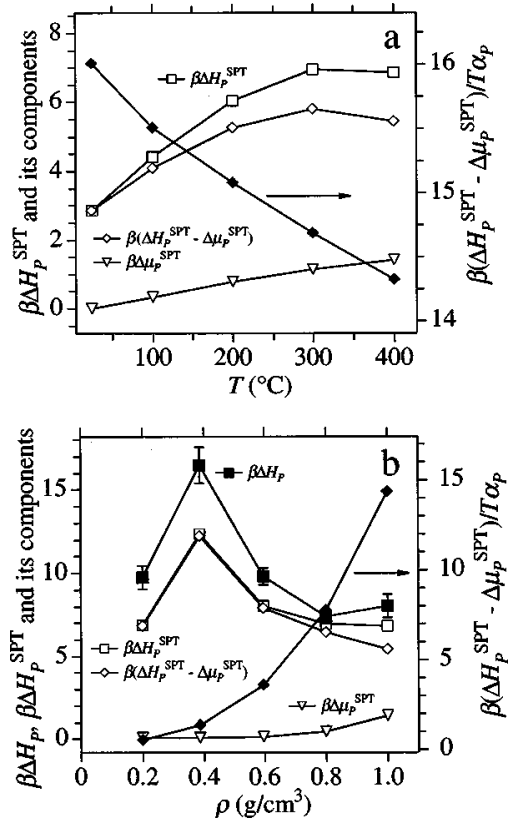

FIG. 18. The excess partial molar enthalpy at constant pressure of the hard sphere solute of exclusion radius $3.0 \AA$ evaluated from the scaled-particle theory. (a) $\beta \Delta H_{P}^{\mathrm{SPT}}$ and its components $\beta \Delta \mu_{P}^{\mathrm{SPT}}$ and $\beta\left(\Delta H_{P}^{\mathrm{SPT}}-\Delta \mu_{P}^{\mathrm{SPT}}\right)$ as functions of the temperature $T$ at the states $A, B, C, D$, and $E$, which involve water densities of $1.0 \mathrm{~g} / \mathrm{cm}^{3} . \beta\left(\Delta H_{P}^{\mathrm{SPT}}-\Delta \mu_{P}^{\mathrm{SPT}}\right) / T \alpha_{P}$ is also shown by the filled symbols. (b) $\beta \Delta H_{P}^{\mathrm{SPT}}$ and its components $\beta \Delta \mu_{P}^{\mathrm{SPT}}$ and $\beta\left(\Delta H_{P}^{\mathrm{SPT}}\right.$ $\left.-\Delta \mu_{P}^{\mathrm{SPT}}\right)$ as functions of the density $\rho$ at the supercritical states $E, F, G, H$, and $I . \beta \Delta H_{P}$ obtained from the simulations and $\beta\left(\Delta H_{P}^{\mathrm{SPT}}-\Delta \mu_{P}^{\mathrm{SPT}}\right) / T \alpha_{P}$ are also shown by the filled symbols.

ameter $\sigma_{v}$ against the temperature, Fig. 18(a) also shows $\beta\left(\Delta H_{P}^{\mathrm{SPT}}-\Delta \mu_{P}^{\mathrm{SPT}}\right) / T \alpha_{P}$, which is equal to the term in the brackets $([\cdots])$ of Eq. $(\mathrm{C} 1)$. It is seen that the reduction in $\sigma_{v}$ at higher temperatures lessens $\beta \Delta H_{P}^{\mathrm{SPT}}$ when the other parameters are fixed. Therefore, Figs. 3(b) and 18(a) imply in the framework of the scaled-particle theory that the temperature dependence of $\beta \Delta H_{P}^{\mathrm{SPT}}$ is determined by that of $T \alpha_{P}$. In this case, a larger $\beta \Delta H_{P}^{\mathrm{SPT}}$ is ascribed to a larger $T \alpha_{P}$. Note in addition that the $T$ dependence of $T \alpha_{P}$ is parallel to that of the "exact" $\beta \Delta H_{P}$ obtained from the simulations, as illustrated in Figs. 3(b) and 11(b). In his original developments of the scaled-particle theory, Pierotti compared the thermodynamics of cavity formation in water and in an organic solvent, and argued that the thermodynamics in the organic solvent is dominated by the enthalpic component because of the large $T \alpha_{P} .^{23,25,38,44,46}$ Our arguments concerning the temperature dependence of the thermodynamics of cavity formation in water are parallel to those by Pierotti. The large enthalpic component in the thermodynamics of cavity formation in high-temperature water is related to the large $T \alpha_{P}$ involved.

We then turn to the density dependence of the excess partial molar enthalpy in the supercritical region. In Fig. 18(b), we show $\beta \Delta H_{P}^{\mathrm{SPT}}$ and its components $\beta \Delta \mu_{P}^{\mathrm{SPT}}$ and $\beta\left(\Delta H_{P}^{\mathrm{SPT}}-\Delta \mu_{P}^{\mathrm{SPT}}\right)$ as functions of the density $\rho$ at a temperature of $400^{\circ} \mathrm{C}$ for a cavity with the radius of $\lambda=3.0 \AA$. This figure also shows, as a reference, the "exact" $\beta \Delta H_{P}$ obtained from the simulations. Since the trend seen in Fig. 18(b) holds over the range of $\lambda$ in Fig. 13(a), the results are shown only at $\lambda=3.0 \AA$ for brevity. It is easy to see that the $\rho$ dependence of $\beta \Delta H_{P}^{\mathrm{SPT}}$ is parallel to that of the "exact" $\beta \Delta H_{P}$ and that both the quantities are not monotonic with respect to the density. The discrepancy between $\beta \Delta H_{P}^{\mathrm{SPT}}$ and $\beta \Delta H_{P}$ arises simply because the optimization of the solvent diameter $\sigma_{v}$ which is made with respect to the excess chemical potential is not perfect. When the components of $\beta \Delta H_{P}^{\mathrm{SPT}}$ are concerned up to a density of $\sim 0.8 \mathrm{~g} / \mathrm{cm}^{3}$, the pressure term $\beta \Delta \mu_{P}^{\mathrm{SPT}}$ makes a minor contribution to the density dependence of $\beta \Delta H_{P}^{\mathrm{SPT}}$. In this region, the density dependence is dominated by the term $\beta\left(\Delta H_{P}^{\mathrm{SPT}}-\Delta \mu_{P}^{\mathrm{SPT}}\right)$. When the density is higher than $\sim 0.8 \mathrm{~g} / \mathrm{cm}^{3}$, the density dependence of $\beta\left(\Delta H_{P}^{\mathrm{SPT}}-\Delta \mu_{P}^{\mathrm{SPT}}\right)$ is compensated by that of $\beta \Delta \mu_{P}^{\mathrm{SPT}}$, and $\beta \Delta H_{P}^{\mathrm{SPT}}$ depends weakly on the density. The effects of the variation in $\sigma_{v}$ and in the packing fraction $y$ can be isolated by examining $\beta\left(\Delta H_{P}^{\mathrm{SPT}}-\Delta \mu_{P}^{\mathrm{SPT}}\right) / T \alpha_{P}$, which is the term in the brackets $([\cdots])$ of Eq. (C1). This term is shown in Fig. 18(b) as a function of $\rho$. It is evident that the term increases monotonically with the density. On the other hand, $T \alpha_{P}$ is a monotonically decreasing function at the densities examined in the present work, as shown in Fig. 4. The density dependence of $\beta \Delta H_{P}^{\text {SPT }}$ up to $\sim 0.8 \mathrm{~g} / \mathrm{cm}^{3}$ is thus determined by the balance between the competing density dependences of $\beta\left(\Delta H_{P}^{\mathrm{SPT}}-\Delta \mu_{P}^{\mathrm{SPT}}\right) / T \alpha_{P}$ and $T \alpha_{P}$. When the density is low $\left(\rho \leqq 0.4 \mathrm{~g} / \mathrm{cm}^{3}\right)$, the increase in the density and the accompanying increase in the solvent diameter $\sigma_{v}$ lead to a larger $\beta \Delta H_{P}^{\text {SPT }}$. When the density is higher $\left(0.4 \mathrm{~g} / \mathrm{cm}^{3} \lesssim \rho \lesssim 0.8 \mathrm{~g} / \mathrm{cm}^{3}\right)$, on the other hand, the density dependence of $\beta \Delta H_{P}^{\mathrm{SPT}}$ is dominated by $T \alpha_{P}$ and the decrease in $T \alpha_{P}$ leads to a smaller $\beta \Delta H_{P}^{\mathrm{SPT}}$. In the high-density region $\left(\rho \geq 0.8 \mathrm{~g} / \mathrm{cm}^{3}\right)$, although $\beta\left(\Delta H_{P}^{\mathrm{SPT}}-\Delta \mu_{P}^{\mathrm{SPT}}\right)$ still reduces with $T \alpha_{P}$, the reduction is compensated by the increase in $\beta \Delta \mu_{P}^{\mathrm{SPT}}$ caused by the pressure elevation.

${ }^{1}$ In this paper, the term "supercritical water" refers to fluid water at a temperature above the critical. Note that the pressure (or density) is not specified.

${ }^{2}$ Water, A Comprehensive Treatise, edited by F. Franks (Plenum, New York, 1972-1982), Vols. 1-7.

${ }^{3}$ J. F. Connolly, J. Chem. Eng. Data 11, 13 (1966).

${ }^{4}$ Z. Alwani and G. Schneider, Ber. Bunsenges. Phys. Chem. 71, 633 (1967).

${ }^{5}$ M. Christoforakos and E. U. Franck, Ber. Bunsenges. Phys. Chem. 90, 780 (1986).

${ }^{6}$ G. Wu, M. Heilig, H. Lentz, and E. U. Franck, Ber. Bunsenges. Phys. Chem. 94, 24 (1990).

${ }^{7}$ R. Deul and E. U. Franck, Ber. Bunsenges. Phys. Chem. 95, 847 (1991).

${ }^{8}$ R. W. Shaw, T. B. Brill, A. A. Clifford, C. A. Eckert, and E. U. Franck, Chem. Eng. News 69, 26 (1991).

${ }^{9}$ J. W. Tester, H. R. Holgate, F. J. Armellini, P. A. Webley, W. R. Killilea, G. T. Hong, and H. E. Barner, in ACS Symposium Series 518, edited by D. W. Tedder and F. G. Pohland (American Chemical Society, Washington DC, 1993).

${ }^{10}$ J. S. Seewald, Nature (London) 370, 285 (1994)

${ }^{11}$ F. N. Spiess et al., Science 207, 1421 (1980).

${ }^{12}$ M. Nakahara, T. Yamaguchi, and H. Ohtaki, Recent Res. Devel. Phys. Chem. 1, 17 (1997).

${ }^{13}$ N. Matubayasi, C. Wakai, and M. Nakahara, Phys. Rev. Lett. 78, 2573, 4309 (1997).

${ }^{14}$ N. Matubayasi, C. Wakai, and M. Nakahara, J. Chem. Phys. 107, 9133 (1997).

${ }^{15}$ N. Matubayasi, C. Wakai, and M. Nakahara, J. Chem. Phys. 110, 8000 (1999).

${ }^{16}$ L. R. Pratt and D. Chandler, J. Chem. Phys. 67, 3683 (1977). 
${ }^{17}$ A. Ben-Naim, Hydrophobic Interactions (Plenum, New York, 1980).

${ }^{18}$ A. Ben-Naim, Solvation Thermodynamics (Plenum, New York, 1987).

${ }^{19}$ L. R. Pratt, Annu. Rev. Phys. Chem. 36, 433 (1985).

${ }^{20}$ D. D. Eley, Trans. Faraday Soc. 35, 1281 (1939).

${ }^{21}$ D. D. Eley, Trans. Faraday Soc. 35, 1421 (1939).

${ }^{22}$ H. S. Frank and M. W. Evans, J. Chem. Phys. 13, 507 (1945).

${ }^{23}$ R. A. Pierotti, J. Phys. Chem. 69, 281 (1965).

${ }^{24}$ F. H. Stillinger, J. Solution Chem. 2, 141 (1973).

${ }^{25}$ R. A. Pierotti, Chem. Rev. 76, 717 (1976).

${ }^{26}$ J. C. Owicki and H. A. Scheraga, J. Am. Chem. Soc. 99, 7413 (1977)

${ }^{27}$ S. Swaminathan, S. W. Harrison, and D. L. Beveridge, J. Am. Chem. Soc. 100, 5705 (1978).

${ }^{28}$ P. J. Rossky and M. Karplus, J. Am. Chem. Soc. 101, 1913 (1979).

${ }^{29}$ A. Geiger, A. Rahman, and F. H. Stillinger, J. Chem. Phys. 70, 263 (1979).

${ }^{30}$ S. Okazaki, K. Nakanishi, H. Touhara, and Y. Adachi, J. Chem. Phys. 71, 2421 (1979)

${ }^{31}$ C. Pangali, M. Rao, and B. J. Berne, J. Chem. Phys. 71, 2982 (1979).

${ }^{32}$ G. Alagona and A. Tani, J. Chem. Phys. 72, 580 (1980).

${ }^{33}$ G. Bolis and E. Clementi, Chem. Phys. Lett. 82, 147 (1981).

${ }^{34}$ W. L. Jorgensen, J. Chem. Phys. 77, 5757 (1982).

${ }^{35}$ D. C. Rapaport and H. A. Scheraga, J. Phys. Chem. 86, 873 (1982).

${ }^{36}$ J. P. M. Postma, H. J. C. Berendsen, and J. R. Haak, Faraday Symp. Chem. Soc. 17, 55 (1982)

${ }^{37}$ W. C. Swope and H. C. Andersen, J. Phys. Chem. 88, 6548 (1984).

${ }^{38}$ B. Lee, Biopolymers 24, 813 (1985).

${ }^{39}$ H. Tanaka, J. Chem. Phys. 86, 1512 (1987).

${ }^{40}$ H. A. Yu and M. Karplus, J. Chem. Phys. 89, 2366 (1988).

${ }^{41}$ A. Pohorille and L. R. Pratt, J. Am. Chem. Soc. 112, 5066 (1990).

${ }^{42}$ B. Guillot, Y. Guissani, and S. Bratos, J. Chem. Phys. 95, 3643 (1991).

${ }^{43}$ L. R. Pratt and A. Pohorille, Proc. Natl. Acad. Sci. USA 89, 2995 (1992).

${ }^{44}$ K. Soda, J. Phys. Soc. Jpn. 62, 1782 (1993).

${ }^{45}$ N. Matubayasi, J. Am. Chem. Soc. 116, 1450 (1994).

${ }^{46}$ M. Ikeguchi, S. Shimizu, S. Nakamura, and K. Shimizu, J. Phys. Chem. B 102, 5891 (1998).

${ }^{47}$ N. Matubayasi and M. Nakahara, J. Chem. Phys. (in preparation).

${ }^{48}$ B. Guillot and Y. Guissani, J. Chem. Phys. 99, 8075 (1993).

${ }^{49}$ R. Crovetto, R. Fernández-Prini, and M. L. Japas, J. Chem. Phys. 76, 1077 (1982).

${ }^{50}$ R. Fernandez-Prini, R. Crovetto, M. L. Japas, and D. Laria, Acc. Chem. Res. 18, 207 (1985).

${ }^{51}$ R. Fernandez-Prini and R. Crovetto, J. Phys. Chem. Ref. Data 18, 1231 (1989).

${ }^{52}$ P. T. Cummings, H. D. Cochran, J. M. Simonson, R. E. Mesmer, and S. Karaborni, J. Chem. Phys. 94, 5606 (1991).

${ }^{53}$ A. A. Chialvo and P. T. Cummings, J. Chem. Phys. 101, 4466 (1994).

${ }^{54}$ P. B. Balbuena, K. P. Johnston, and P. J. Rossky, J. Am. Chem. Soc. 116, 2689 (1994).

${ }^{55}$ P. B. Balbuena, K. P. Johnston, and P. J. Rossky, J. Phys. Chem. 99, 1554 (1995).

${ }^{56}$ L. W. Flanagin, P. B. Balbuena, K. P. Johnston, and P. J. Rossky, J. Phys. Chem. 99, 5196 (1995).

${ }^{57}$ A. A. Chialvo, P. T. Cummings, H. D. Cochran, J. M. Simonson, and R. E. Mesmer, J. Chem. Phys. 103, 9379 (1995).

${ }^{58}$ A. A. Chialvo and P. T. Cummings, J. Phys. Chem. 100, 1309 (1996).

${ }^{59}$ P. B. Balbuena, K. P. Johnston, and P. J. Rossky, J. Phys. Chem. 100, 2706 (1996)

${ }^{60}$ P. B. Balbuena, K. P. Johnston, and P. J. Rossky, J. Phys. Chem. 100, 2716 (1996).

${ }^{61}$ P. B. Balbuena, K. P. Johnston, P. J. Rossky, and J. K. Hyun, J. Phys. Chem. B 102, 3806 (1998).

${ }^{62}$ A. A. Chialvo, P. T. Cummings, J. M. Simonson, and R. E. Mesmer, J. Chem. Phys. 110, 1064 (1999).

${ }^{63}$ H. Sato and F. Hirata, J. Phys. Chem. B 103, 6596 (1999).

${ }^{64}$ J. Gao, J. Am. Chem. Soc. 115, 6893 (1993).

${ }^{65}$ D. M. Pfund, J. G. Darab, J. L. Fulton, and Y. Ma, J. Phys. Chem. 98, 13102 (1994).

${ }^{66}$ J. C. Wheeler, Ber. Bunsenges. Phys. Chem. 76, 308 (1972).

${ }^{67}$ R. F. Chang and J. M. H. Levelt Sengers, J. Phys. Chem. 90, 5921 (1986).

${ }^{68}$ J. V. Sengers and J. M. H. Levelt Sengers, Annu. Rev. Phys. Chem. 37, 189 (1986)

${ }^{69}$ P. G. Debenedetti and S. K. Kumar, AIChE J. 34, 645 (1988).

${ }^{70}$ A. A. Chialvo and P. T. Cummings, AIChE J. 40, 1558 (1994).

${ }^{71}$ S. C. Tucker and M. W. Maddox, J. Phys. Chem. B 102, 2437 (1998).
${ }^{72}$ O. Kajimoto, Chem. Rev. 99, 355 (1999).

${ }^{73}$ N. Matubayasi, L. H. Reed, and R. M. Levy, J. Phys. Chem. 98, 10640 (1994).

${ }^{74}$ N. Matubayashi and R. M. Levy, J. Phys. Chem. 100, 2681 (1996).

${ }^{75}$ N. Matubayasi, E. Gallicchio, and R. M. Levy, J. Chem. Phys. 109, 4864 (1998).

${ }^{76}$ M. P. Allen and D. J. Tildesley, Computer Simulation of Liquids (Oxford University Press, Oxford, 1987).

${ }^{77}$ B. Widom, J. Chem. Phys. 39, 2808 (1963).

${ }^{78}$ K. S. Shing and S. T. Chung, J. Phys. Chem. 91, 1674 (1987).

${ }^{79}$ T. C. Beutler, D. R. Béguelin, and W. F. van Gunsteren, J. Chem. Phys. 102, 3787 (1995).

${ }^{80}$ P. E. Smith, J. Phys. Chem. B 103, 525 (1999).

${ }^{81}$ N. Yoshii, H. Yoshie, S. Miura, and S. Okazaki, J. Chem. Phys. 109, 4873 (1998).

${ }^{82}$ H. J. C. Berendsen, J. R. Grigera, and T. P. Straatsma, J. Phys. Chem. 91, 6269 (1987)

${ }^{83}$ Y. Guissani and B. Guillot, J. Chem. Phys. 98, 8221 (1993).

${ }^{84}$ M. Sprik and M. L. Klein, J. Chem. Phys. 89, 7556 (1988).

${ }^{85}$ P. Ahlström, A. Wallqvist, S. Engström, and B. Jönsson, Mol. Phys. 68, 563 (1989).

${ }^{86}$ P. Cieplak, P. Kollman, and T. Lybrand, J. Chem. Phys. 92, 6755 (1990).

${ }^{87}$ L. X. Dang, J. Chem. Phys. 97, 2659 (1992).

${ }^{88}$ A. Wallqvist and B. J. Berne, J. Phys. Chem. 97, 13841 (1993).

${ }^{89}$ E. S. Fois, M. Sprik, and M. Parrinello, Chem. Phys. Lett. 223, 411 (1994).

${ }^{90}$ D. N. Bernardo, Y. Ding, K. Krogh-Jespersen, and R. M. Levy, J. Phys. Chem. 98, 4180 (1994).

${ }^{91}$ S. W. Rick, S. J. Stuart, and B. J. Berne, J. Chem. Phys. 101, 6141 (1994).

${ }^{92}$ R. D. Mountain, J. Chem. Phys. 103, 3084 (1995).

${ }^{93}$ A. Famulari, R. Specchio, M. Sironi, and M. Raimondi, J. Chem. Phys. 108, 3296 (1998).

${ }^{94}$ B. D. Bursulaya and H. J. Kim, J. Chem. Phys. 110, 9646 (1999).

${ }^{95}$ H. Sato and F. Hirata, J. Chem. Phys. 111, 8545 (1999).

${ }^{96}$ When the system is near the critical point, the correlation between molecules in the system is long ranged and a large unit cell is required for a simulation. According to Sengers and Levelt Sengers expression, the correlation length of water is estimated to be $\sim 15 \AA$ at the critical density when the temperature is $400{ }^{\circ} \mathrm{C}$ (Ref. 68). Among the thermodynamic states shown in Table I, the states $H$ and $I$ are expected to involve large correlation lengths. The correlation lengths at these states will be less than $\sim 15 \AA$, however, since the densities of the states $H$ and $I$ are not the critical. Therefore, since the average lengths of the cubic unit cells are $\sim 37$ and $\sim 46 \AA$ for the states $H$ and $I$, respectively, they are at least $\sim 2.5$ times as large as the corresponding correlation lengths.

${ }^{97}$ Henry's constant is given by $\rho k_{B} T \exp (\beta \Delta \mu)$, where $\rho$ is the solvent density. It is shown, however, that Henry's constant and its density and temperature derivatives are not convenient to extract the solute-induced effects (Refs. 17, 18, and 48)

${ }^{98}$ D. E. Smith and A. D. J. Haymet, J. Chem. Phys. 98, 6445 (1993).

${ }^{99}$ In the isothermal-isobaric ensemble, $\exp \left(-\Delta \mu / k_{B} T\right)$ is equal to $p(\lambda) V(\lambda) / V(0)$, where $V(\lambda)$ is the average volume of the system when a hard sphere solute of exclusion radius $\lambda$ is successfully inserted. Obviously, $V(0)$ is the average volume of the pure solvent system. When the system is large enough, $V(\lambda) / V(0)$ approaches unity and the ensemble dependence of $p(\lambda)$ vanishes. In this paper, we actually calculate $p(\lambda) V(\lambda) / V(0)$ and identify it with $p(\lambda)$.

${ }^{100}$ H. J. C. Berendsen, J. P. M. Postma, W. F. van Gunsteren, and J. Hermans, in Intermolecular Forces, edited by B. Pullmann (Reidel, Dordrecht, 1981).

${ }^{101}$ R. D. Mountain, J. Chem. Phys. 90, 1866 (1989).

${ }^{102}$ T. I. Mizan, P. E. Savage, and R. M. Ziff, J. Phys. Chem. 98, 13067 (1994).

${ }^{103}$ G. Löffler, H. Schreiber, and O. Steinhauser, Ber. Bunsenges. Phys. Chem. 98, 1575 (1994).

${ }^{104}$ A. G. Kalinichev and J. D. Bass, Chem. Phys. Lett. 231, 301 (1994).

${ }^{105}$ J. Brodholt, M. Sampoli, and R. Vallauri, Mol. Phys. 86, 149 (1995).

${ }^{106}$ A. G. Kalinichev and J. D. Bass, J. Phys. Chem. A 101, 9720 (1997).

${ }^{107}$ P. Jedlovszky et al., J. Chem. Phys. 108, 8528 (1998).

${ }^{108}$ The International Association for the Properties of Water and Steam, IAPWS Formulation 1995, Fredericia, Denmark, 1996.

${ }^{109}$ In the ideal gas limit, both $\rho k_{B} T \kappa_{T}$ and $T \alpha_{P}$ are equal to unity. In this 
sense, $\rho k_{B} T \kappa_{T}$ and $T \alpha_{P}$ are the normalized forms of $\kappa_{T}$ and $\alpha_{P}$, respectively, relative to the corresponding ideal gas limits.

${ }^{110}$ Even when the solute polarizability is included, its induced attraction with water is weaker at higher temperatures. Thus, the inclusion of the solute polarization actually widens the range of thermodynamic states at which the excess chemical potential of a nonpolar solute is larger than that at the ambient state.

${ }^{111}$ R. D. Mountain and A. Wallqvist, NISTIR, 5778 (1996).

${ }^{112}$ In Ref. 111, it has been shown for SPC/E water that the dielectric constant is smaller when the density is lower and/or the temperature is higher. While the dipole moment of a water molecule is fixed in the SPC/E model, it is to be described as a function of the density and temperature for a more realistic simulation of water. However, even when the state-dependent nature of the dipole moment is incorporated into the interaction potential model of water, the dipole moment should be smaller at lower densities and/or higher temperatures (this is indeed valid in our effective model presented in Ref. 15). Therefore, the dielectric constant is smaller at lower densities and/or higher temperatures also for a water potential model with a state-dependent dipole moment since the dielectric constant is smaller for a smaller dipole moment at given density and temperature.

${ }^{113}$ In Ref. 48, Guillot and Guissani called $\left\langle u_{\mathrm{uv}}\right\rangle$ and $\left(\Delta \mu-\left\langle u_{\mathrm{uv}}\right\rangle\right)$ the energetic and entropic components, respectively, on the basis of Yu and Karplus's arguments concerning the cancellation of the solvent reorganization term (Ref. 40). As pointed out by Lee, however, the discussion about the cancellation is rather semantic (Ref. 114). Actually, the solvent reorganization is shown to make a nonzero contribution to $\Delta \mu$ when $\Delta \mu$ is expressed in terms of the multiparticle correlation functions at the initial and final states (note that only the information at the initial and final states is available for a real system) (Refs. 73 and 75). Thus, we do not follow Guillot and Guissani, and call $\left\langle u_{\mathrm{uv}}\right\rangle$ and $\left(\Delta \mu-\left\langle u_{\mathrm{uv}}\right\rangle\right)$ the interaction and cavity components, respectively.

${ }^{114}$ B. Lee, Biophys. Chem. 51, 271 (1994).

${ }^{115}$ In the text, the interaction component is simply defined as the average solute-solvent interaction $\left\langle u_{\mathrm{uv}}\right\rangle$. An alternative definition of the interaction component may be given on the basis of the Weeks-ChandlerAndersen (WCA) scheme to decompose the solute-solvent interaction into the attractive and repulsive parts (Ref. 116). In this case, the interaction component is taken as the average sum of the WCA attractive interaction of methane with all the water molecules, and the cavity component is equal to the difference between $\Delta \mu$ and the interaction component. The trend concerning the decomposition into the interaction and cavity components described in Sec. IV C is still valid when the components are defined on the basis of the WCA scheme.

${ }^{116}$ J. D. Weeks, D. Chandler, and H. C. Andersen, J. Chem. Phys. 54, 5237 (1971).

${ }^{117}$ When $\lambda$ is sufficiently small, the probability of finding a cavity of radius $\lambda$ is determined only by the density and the corresponding $\beta \Delta \mu$ is independent of the temperature. When $\lambda$ is large enough, $\beta \Delta \mu$ is proportional to $P / T$ and exhibits a strong temperature dependence according to Table I.

${ }^{118}$ R. D. Mountain, J. Chem. Phys. 110, 2109 (1999).

${ }^{119}$ In Ref. 79, Beutler et al. found that the excess chemical potential is quadratic with respect to the thermal radius of the solute-solvent repulsive core. When $\Delta \mu(\sigma)$ is assumed to be quadratic in $\sigma,\langle\partial u(\sigma) / \partial \sigma\rangle_{\sigma}$ in Fig. 17 is linear with respect to $\sigma$. In this case, Fig. 17 gives that $\Delta \mu\left(\sigma_{\mathrm{Me}-\mathrm{O}}\right)=2.85 \pm 1.00$ and $10.73 \pm 0.39 \mathrm{kcal} / \mathrm{mol}$ at the states $A$ and $E$, respectively. 